\title{
QUEEN'S UNIVERSITY
}

\section{POLYMERS RESEARCH GROUP}

19 Division Street, Kingston, ON, K7L 3N6 Canada

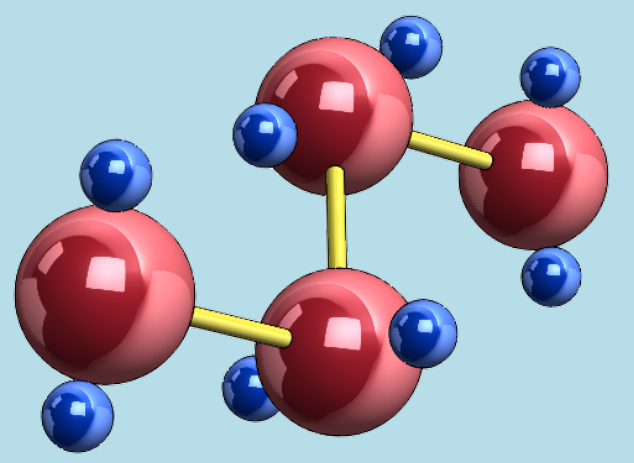

\section{DIE DROOL AND POLYMER DEGRADATION}

Geoffrey S. Hoy ${ }^{1}$, A. Jeffrey Giacomin ${ }^{*, 1,2}$ and Peter H. Gilbert ${ }^{1}$

${ }^{1}$ Chemical Engineering Department, Polymers Research Group, Queen's University, Kingston, Ontario, CANADA K7L 3N6

${ }^{2}$ Mechanical and Materials Engineering Department, Queen's University, Kingston, Ontario, CANADA K7L 3N6

This report is circulated to persons believed to have an active interest in the subject matter; it is intended to furnish rapid communication and to stimulate comment, including corrections of possible errors.

\footnotetext{
*Corresponding author (giacomin@queensu.ca)
} 


\title{
DIE DROOL AND POLYMER DEGRADATION
}

\author{
Geoffrey S. Hoy ${ }^{1}$, A. Jeffrey Giacomin ${ }^{\star, 1,2}$ and Peter H. Gilbert ${ }^{1}$ \\ ${ }^{1}$ Chemical Engineering Department, Polymers Research Group, \\ Queen's University, Kingston, Ontario, CANADA K7L 3N6 \\ ${ }^{2}$ Mechanical and Materials Engineering Department, \\ Queen's University, Kingston, Ontario, CANADA K7L 3N6
}

\begin{abstract}
Die drool is an operational problem associated with polymer extrusion. Extrudate collects outside of the die, necessitating periodic disruptions for cleaning. There exists some debate as to the mechanism that produces die drool: stress induced fractionation or thermal degradation. This paper examines the latter. In cohesive failure, a slip discontinuity develops in the velocity profile, where heat is generated by friction. This slip heating can contribute to resin degradation, resulting in lower molecular weight fragments in the die drool. This paper examines the kinetics of this degradation, its influence on die drool rates, and on the resulting polymer concentration profiles in the drool layer and in the bulk.

Keywords: Die drool, slip heating, cohesive slip, polymer degradation, scission
\end{abstract}

*Corresponding author (giacomin@queensu.ca) 


\section{CONTENTS}

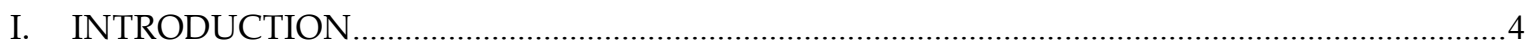

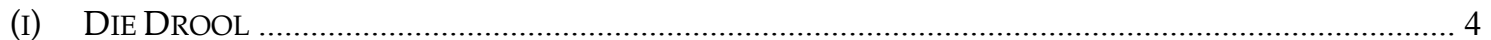

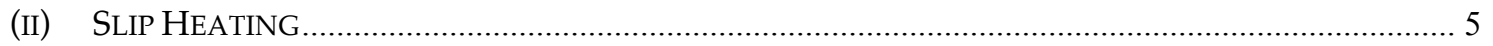

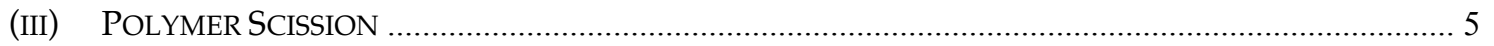

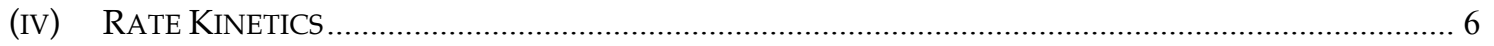

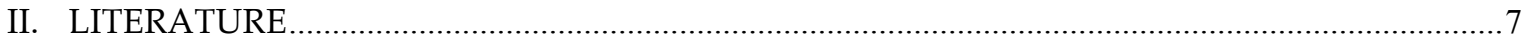

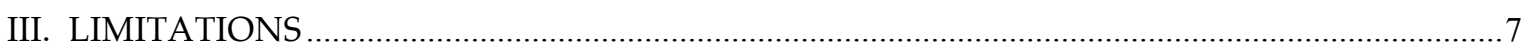

IV. TEMPERATURE INDEPENDENT CHAIN SCISSION KINETICS …..........................................

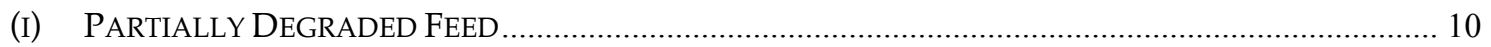

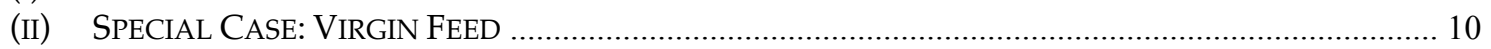

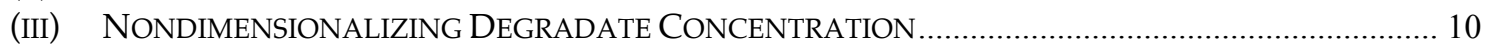

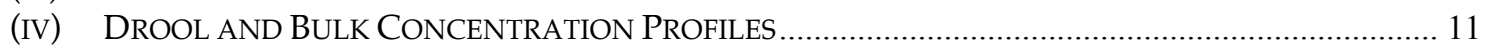

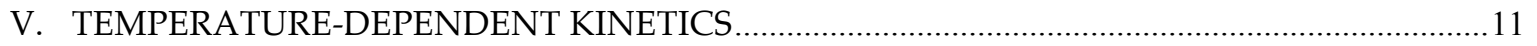

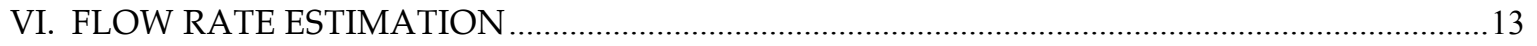

(I) SimplificAtion: CONSTANT AVERAGE CONCENTRATION THROUGH TAYLOR SERIES ................. 14

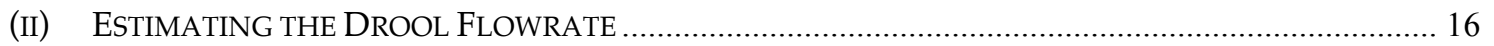

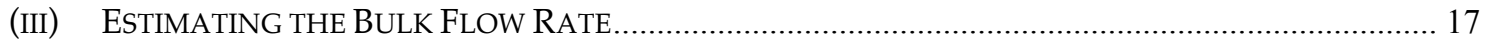

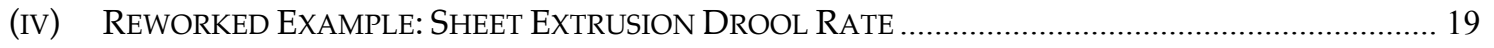

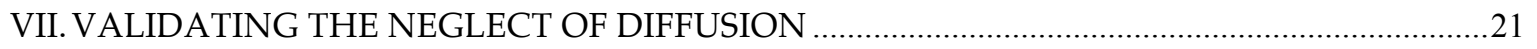

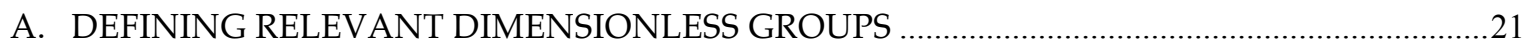

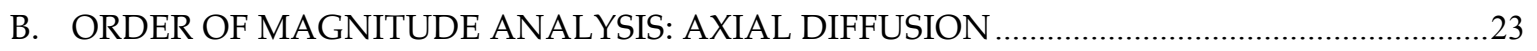

C. ORDER OF MAGNITUDE ANALYSIS: TRANSVERSE DIFFUSION ……...................................24

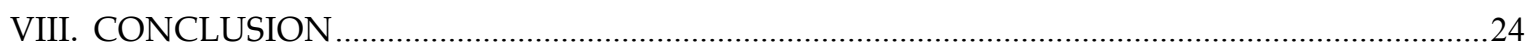

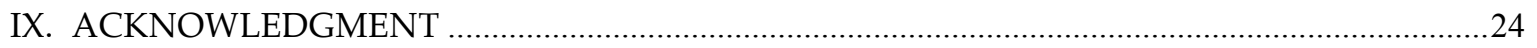

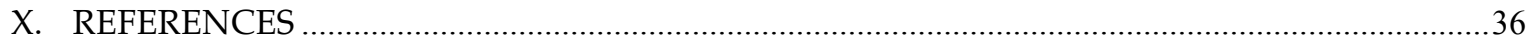




\section{TABLES}

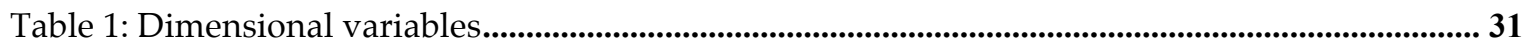

Table 2: Dimensionless variables. .......................................................................................................................... 33

Table 3: Literature relevant to die drool with degradation.......................................................................... 35

\section{FIGURES}

Figure 1: Flow profile for proposed cohesive slip creation of die drool [15]......................................... 25

Figure 2: Velocity profiles and concomitant steady state temperature profiles before adjustment for concentration effects on viscosity $[15] \ldots \ldots+\cdots \cdots \cdots$

Figure 3: Dimensionless drool concentration profile from the slip interface to the wall ................... 27

Figure 4: Dimensionless bulk concentration profile from the slip interface to the midplane of the

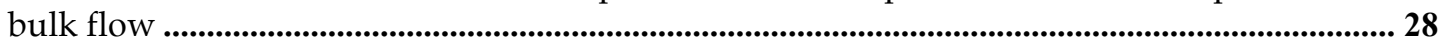

Figure 5: Dimensionless drool velocity profiles from the wall to the slip interface........................... 29

Figure 6: Dimensionless bulk velocity profile from the slip interface to the midplane of the bulk flow ......................................................................................................................................................... 30 


\section{INTRODUCTION}

This paper extends a model for die drool and the resulting slip heating [1] by exploring the role of degradation within the drool layer. We incorporate current theory of die drool mechanics, rheology, tribology and polymer scission kinetics.

\section{(i) Die Drool}

Die drool is the accumulation of molten plastic on the open face of an extruder die [1]. Die drool affects the quality of the extruded product; for example, die drool can scratch the extrudate, creating die lines [2]. The appearance of die drool also lowers productivity, as production must be shut down to remove the drool from the die face [3].

Die drool has been attributed to external mechanical forces and internal rheology. External die drool generates small flakes or powder in a very short time frame (seconds-minutes), whereas the internal die drool accumulates in compact shapes over a longer time frame (tens of minutes to hours) [4]. Mechanically, external die drool has been attributed to negative (suction) pressure that occurs in the die exit edges (see Figure 1) [4]. External die drool formation can be mitigated through proper extruder and die design, specifically through die geometry $[5,6]$, die coatings or die material selection [7]. This external die drool formation has been proven to be independent of die swell, even though both swell and drool are dependent on similar properties [8]. The formation of internal die drool has been hypothesized to be the result of:

\section{Molar polymer weight fractionation}

Shear induced fractionation could cause low molecular weight material to migrate to the die wall [9]. As a result, the high stresses near the exit may cause this lower molecular weight material to separate from the extrudate and accumulate on the die surface [10]. This theory has been supported experimentally; gel permeation chromatography [11] and static laser light scattering [12] have demonstrated that die drool has a markedly different composition than the bulk extrudate. Theoretically, models have supported these experimental findings [13]. The die drool exhibits a larger fraction of lower molecular weight polymer. The actual source of this lower molecular weight material is widely contested; when this fractionation is induced artificially in experimental apparatuses, the time frame for fractionation is orders of magnitude longer than the on-stream time of an extruder (say, 100 to 1000 hours) [14].

\section{Stress-induced cohesive chain disentanglement (slip) theory}

The fluid slips on itself creating a drool layer and a bulk fluid layer, separated by a theoretical boundary defined by a step discontinuity in the fluid velocity profile (Figure 1) [15]. In other words, the polymer chains are not entangled across this layer, and the bulk and drool flow can be considered separately. This cohesive slip layer (drool layer) will emerge from the die and collect on the exterior of the die, forming the die drool. This theory, similar to the fractionation theory, is not exclusive of the adhesive failure mechanism (wall slip) [4]. The fractionation of the resulting die drool has been proposed to the result of thermal degradation, caused by slip heating at the cohesive slip layer. 
Schmalzer and Giacomin derived the drool rate relative to the overall bulk fluid flow rate. This relationship is called the buildup ratio [1,15].

$$
B R \equiv \frac{Q_{d}}{Q_{b}}
$$

which we will use throughout this work.

\section{(ii) Slip Heating}

According to cohesive slip theory, the step discontinuity in the velocity profile will give rise to frictional heating, also called slip heating. The slip velocity is the difference between the velocity of the bulk and drool at the cohesive slip interface $[16,17,18,19]$.

$$
v_{s}=v_{b}-v_{d}
$$

The drool layer thickness is a function of pressure drop in the extruder and of cohesive fracture strength:

$$
d=\frac{h}{2}+\frac{\tau_{c} L}{\Delta P}
$$

The dimensionless form of the cohesive fracture strength is always unity regardless of the values of $\tau_{c}, \Delta P$, and $L$. This is a result of the dependence of the bulk height, $b$, on $\Delta P, L$, and $\tau_{c}$ [16]:

$$
\sigma_{c}=\frac{\tau_{c} L}{b \Delta \mathrm{P}}
$$

The magnitude of the frictional heat flux is the product of the shear stress and slip speed [16]:

$$
q_{x}=\left.v_{s} \tau_{x y}\right|_{x=0}
$$

This theory supposes that the shear stress at this slip boundary is material specific, and is referred to as the cohesive fracture strength, $\tau_{c}$. The slip heating of a fluid in an extruder is a function of this heat flux and the distance between the wall and the slip interface. The steady state temperature profiles (see Figure 1) of a Newtonian fluid (drool and bulk, respectively) in a slit extruder has been derived from the equation of energy (Eq. B.8-1 of [20]), assuming negligible convective heat transfer [16].

$$
\begin{aligned}
& \Theta_{d, \infty}(\zeta)=F(\zeta+a)+\Theta_{w} ;-a \leq \zeta \leq 0 \\
& \Theta_{b, \infty}(\zeta)=F a+\Theta_{w} ; 0 \leq \zeta \leq 1
\end{aligned}
$$

which we will use below.

\section{(iii) Polymer Scission}

Die drool contains significantly more low molecular weight material than the virgin resin [21]. Additionally, spiking polyethylene film with low molecular weight polyethylene wax accelerates drool [10, 22, 23]. In these spiking 
experiments, we observe a power-law relationship of buildup ratio with polydispersity (Eq. (25) of [10]):

$$
B R \cong \alpha_{0}\left(\frac{M_{\mathrm{w}}}{M_{n}}\right)^{\beta}
$$

This suggests that, if low molecular weight material is produced upstream of an extrusion die, and specifically, through chain scission, then accelerated drool is likely to result. Assuming fractionation is negligible, this abundance of low molecular weight material implies that the polymer undergoes scission during the drool formation process. Polymer chains under high temperatures undergo this degradation reaction, splitting to form smaller sub-unit chains. The simplest model that describes scission assumes that a polymer, A, will degrade into two chains, B, which are exactly half of original molecular weight.

$$
A \rightarrow 2 B
$$

In practice, the molecular weight of a polymer extrusion will vary. This range of molecular weights is described by the molecular weight distribution (MWD) or, more concisely, with the polydispersity index [3].

\section{(iv) Rate Kinetics}

Rate kinetics dictate the reaction rate. Evolving concentration profiles can be constructed from rate kinetics data, where the extent of reaction varies as the reaction progresses. In an extruder, the time dependence (or time on-stream) of the reaction progression can be expressed in terms of position within the flow field and velocity. For slit flow:

$$
t_{s}(y)=\frac{y}{v_{y}(x)}
$$

where Figure 1 and Figure 2 illustrate the slit flow, and Table 1 and Table 2 define the variables.

The dimensionless steady state velocity profiles for the bulk and drool flows have been developed for cohesive slip in a slit die [18]:

$$
\begin{aligned}
& \varphi_{d}(\zeta)=\frac{a}{\ln ^{2} \eta_{i, \infty}}\left[\eta_{i, \infty}^{-\left(1+\frac{\xi}{a}\right)} a+\eta_{i, \infty}^{-\left(1+\frac{\xi}{a}\right)} \ln \eta_{i, \infty}\left(\zeta-\sigma_{C}\right)-a+\ln \eta_{i, \infty}\left(\sigma_{C}+a\right)\right] ;-a \leq \zeta \leq 0 \\
& \varphi_{b}(\zeta)=-\frac{1}{2 \eta_{b}} \zeta^{2}+\frac{1}{\eta_{b}} \zeta+\frac{a}{\ln ^{2} \eta_{i, \infty}}\left[\frac{a-\sigma_{C} \ln \eta_{i, \infty}}{\eta_{i, \infty}}-a+\ln \eta_{i, \infty}\left(\sigma_{C}+a\right)\right]+\varphi_{S} ; 0 \leq \zeta \leq 1
\end{aligned}
$$

Reaction rates depend on the component concentrations and on rate constants. The simplest kinetics expression for the decomposition of a polymer, $A$, is the irreversible first order reaction [24].

$$
\frac{d c_{B}}{d t}=-2 \frac{d c_{A}}{d t}=k c_{A}
$$


whose first order rate constant, $k$, depends on temperature, and this dependence is normally given by the Arrhenius equation [24]:

$k=A e^{\frac{-E a}{R T}}$

which we will use below.

\section{LITERATURE}

The die drool phenomenon has been widely studied, but the theories vary widely for its cause. This paper addresses the internal causes of die drool and is independent of previous papers addressing extruder and die design, as per Ding et al. [2], Musil et al. [6] and Zatloukal et al. [25]. Section I summarizes current literature on internal die drool theory and slip heating, and Table 3 classifies and compares these papers.

\section{LIMITATIONS}

This paper will build on a previously constructed solution [18, 37]. The fluid examined in this paper is Newtonian, and convective heat transfer has been neglected. Moreover, our solution neglects diffusion of the low molecular weight species generated by the polymer scission, and the solution for slip heating was developed for a monodisperse polymer. As soon as the polymer degrades, a concentration gradient develops. The diffusion of the species B through A would add another layer of complexity to the proposed solution. We address this diffusion with the equation of continuity for a binary system (Eq. B.11-2 of [20]):

$$
\rho\left(\frac{\partial \omega_{B}}{\partial t}+v_{x} \frac{\partial \omega_{B}}{\partial x}+v_{y} \frac{\partial \omega_{B}}{\partial y}+v_{z} \frac{\partial \omega_{B}}{\partial z}\right)=\rho \mathscr{D}_{B A}\left[\frac{\partial^{2} \omega_{B}}{\partial x^{2}}+\frac{\partial^{2} \omega_{B}}{\partial y^{2}}+\frac{\partial^{2} \omega_{B}}{\partial z^{2}}\right]+r_{B}
$$

See Section VII below for an order of magnitude analysis of diffusion.

We neglect the variations in the scission reactions that could arise from the slip heating, and we attempt to address this factor at the most elementary level. However, in practice, the following five factors could complicate scission:

\section{Polydispersity}

The heterogeneity of chain lengths will affect the rheological and reaction analysis. Further, polydispersity has itself been linked to the die lip buildup ratio [see Eq. (8)].

\section{Uneven Split}

Though it is unlikely that the resulting polymers would be exactly half of the initial length [26], scission near the chain ends (less than one full entanglement length from the end is far less likely than anywhere else. We do expect, however, that on average, the single-step scission simplification in a molar average sense will be accurate, so long as, scission stops with the initial step [see Eq. (19)]. There is the potential for other reactions, such as crosslinking between the degraded polymer chains, which has been observed experimentally [32]. Of course, in 
many polymer chemistries, the production of radicals is a necessary condition for crosslinking.

\section{Heat of Degradation}

The thermal scission of polymers can generate or consume heat. The endo/exothermicity of scission is material chemistry dependent [27]. Since scission is generally a low-concentration event, we do not expect this to matter. However, we have yet to carry out a proper transport phenomena analysis of this.

\section{Effect of Degradation on Cohesive Slip Strength}

Theory predicts that slip velocity will vary with the miscibility of the polymers present in the mixture [28]. With the generation of new polymer products, the slip heating term derived to approximate the thermal degradation will also change. This effect on the slip heating has been considered negligible so long as the concentration of the smaller chain polymer, $B$, is insignificant when compared to the polymer, $A$.

\section{Low Molecular Weight}

Shear thinning behavior complicates die drool, but our analysis is restricted to polymeric systems whose molecular weights fall below the entanglement molecular weight (such as commercial grades of condensation polymers such as nylon, polyester and polycarbonate).

\section{TEMPERATURE INDEPENDENT CHAIN SCISSION KINETICS}

We approximate polymer scission stoichiometry as:

$$
A \rightarrow 2 B
$$

so that:

$$
\frac{d c_{B}}{d t}=-2 \frac{d c_{A}}{d t}
$$

where each polymer molecule splits in half, and if there is no low molecular weight polymer in the feed (special case):

$$
C \equiv c_{A}+\frac{1}{2} c_{B} ; c_{B 0}=0
$$

Polymer scission into two equal chains only happens in a number averaging sense. Stoichiometry given by Eq. (16) also neglects further scission:

$B \rightarrow 2 C$

If there is low molecular weight polymer $(B)$ in the feed (general solution):

$C \equiv c_{A 0}+c_{B 0}=c_{A}+\frac{1}{2} c_{B}+c_{B 0}, c_{B 0} \neq 0$

We have defined a system with a first-order reaction, whose degradate production rate can be described in terms of the high molecular weight material, $A$ :

$$
\frac{d c_{B}}{d t}=k c_{A}
$$


By degradate, we mean the degradation product, $B$, in Eq. (16). In a flowing degrading system, such as plastics extrusion, the relevant time $t$ is time on-stream of the high molecular weight reactant (not to be confused with its residence time or of the extruder run time). Time on-stream differs from streamline to streamline, and in extrusion dies, time on-stream increases near no-slip boundaries. Absent wall slip, the time on-stream at the wall is infinite. In this paper, we consider only the steady state solution, and thus, we express time on-stream as:

$$
t=\frac{y}{v_{y}(x)}
$$

Replacing the differential time in Eq. (21) with the expression from Eq. (22) and rearranging yields a degradate concentration gradient as a function of velocity, and position rather than time:

$$
\frac{d c_{B}}{d y}=\frac{k c_{A}}{v_{y}(x)}
$$

which holds when diffusion can be neglected. See Section VII below for an order of magnitude analysis of diffusion. Were diffusion important, the concentration gradients diminish, and thus:

$$
\frac{d c_{B}}{d y}<\frac{k c_{A}}{v_{y}(x)}
$$

The concentration of $c_{A}$ can be expressed as a function of the virgin resin concentration, $C_{A 0}$, and the localized degraded resin concentration, $C_{B}$ :

$$
C_{A 0}=C_{A}+\frac{1}{2} C_{B}
$$

Rearranging Eq. (23), and replacing $c_{A}$ in Eq. (25) yields:

$$
\frac{d c_{B}}{d y}=\frac{k\left(c_{A 0}-\frac{1}{2} c_{B}\right)}{v_{y}(x)}
$$

which can be rearranged to:

$$
\frac{d c_{B}}{d y}=\frac{k}{v_{y}(x)} c_{A 0}-\frac{k}{2 v_{y}(x)} c_{B}
$$

which has general form:

$$
\frac{d y}{d x}=C_{1}-C_{2} y
$$

which has general solution:

$$
y=\frac{C_{1}}{C_{2}}+C_{3} e^{-C_{2} x}
$$

and thus, for our problem:

$$
C_{1}=\frac{k c_{A 0}}{v_{y}(x)}
$$


and:

$C_{2}=\frac{k}{2 v_{y}(x)}$

and hence:

$C_{B}=2 c_{A 0}+C_{3} e^{-\frac{k}{2 v_{y}(x)} y}$

which we will use below.

\section{(i) Partially Degraded Feed}

Using Eq. (32), an alternative constant can be defined for the new boundary conditions. To find $C_{3}$, we can use the entry condition, $y=0$, where the concentration of $C_{B}$ is now $C_{B 0}$, by definition. This condition can be substituted into Eq. (30) to yield:

$c_{B 0}=2 c_{A 0}+C_{3} e^{-\frac{k}{2 v_{y}(x)}(0)}$

which simplifies to:

$C_{3}=c_{B 0}-2 c_{A 0}$

which, when combined with Eq. (32), gives:

$c_{B}=2 c_{A 0}+\left(c_{B 0}-2 c_{A 0}\right) e^{-\frac{k}{2 v_{y}(x)} y}$

which we will use below.

\section{(ii) Special Case: Virgin Feed}

For the special case of undegraded feed, the entry $(y=0)$ condition is $c_{B}=0$. Substituting this condition into Eq. (35) gives:

$c_{B}=2 c_{A 0}-2 c_{A 0} e^{\frac{-k}{2 v_{y}(x)} y}$

\section{(iii) Nondimensionalizing Degradate Concentration}

Eq. (36) can be nondimensionalized using Table 2 of Gilbert and Giacomin [37] and by defining the dimensionless concentration:

$\Upsilon \equiv \frac{c_{B}}{c_{A 0}}$

and the dimensionless inlet resin purity index:

$g \equiv \frac{c_{B 0}}{c_{A 0}}$

Inserting Eq. (37) and (38) into (36) yields:

$\Upsilon(\zeta, \varsigma)=2+(g-2) e^{-\frac{\epsilon}{2} \frac{\zeta}{\varphi_{\varsigma}(\zeta)}}$

which we will use below. 


\section{(iv) Drool and Bulk Concentration Profiles}

The profile given by Eq. (39) is function of the velocity in the $y$-direction. There are four boundaries in this cohesive slip model: two for no wall slip, and two for the cohesive slip boundaries. Gilbert and Giacomin [37] have derived two dimensionless expressions from this model, one for the drool layer:

$$
\varphi_{d}(\zeta)=\frac{a}{\ln ^{2} \eta_{i, \infty}}\left[\eta_{i, \infty}^{-\left(1+\frac{\xi}{a}\right)} a+\eta_{i, \infty}^{-\left(1+\frac{\xi}{a}\right)} \ln \eta_{i, \infty}\left(\zeta-\sigma_{C}\right)-a+\ln \eta_{i, \infty}\left(\sigma_{C}+a\right)\right] ;-a \leq \zeta \leq 0
$$

and another for the bulk:

$$
\varphi_{b}(\zeta)=-\frac{1}{2 \eta_{b}} \zeta^{2}+\frac{1}{\eta_{b}} \zeta+\frac{a}{\ln ^{2} \eta_{i, \infty}}\left[\frac{a-\sigma_{C} \ln \eta_{i, \infty}}{\eta_{i, \infty}}-a+\ln \eta_{i, \infty}\left(\sigma_{C}+a\right)\right]+\varphi_{S} ; 0 \leq \zeta \leq 1
$$

Our model will also require four boundary conditions: two for the walls, where the mole fraction of B will approach unity, and two at the cohesive slip boundaries, defined by the step discontinuity in the velocity profile $\varphi_{s}$ :

$$
\Upsilon_{s}(0, \varsigma)=2+(g-2) \exp \left(-\frac{\epsilon}{2 \varphi_{s}} \varsigma\right)
$$

The concentration in the drool layer is thus given by:

$$
\Upsilon_{d}(\zeta, \varsigma)=2+(g-2) \exp \left(-\frac{\epsilon}{2} \frac{\zeta}{\varphi_{d}(\zeta)}\right)
$$

Inserting Eq. (40) into Eq. (43), we find:

$$
\Upsilon_{d}(\zeta, \varsigma)=2+(g-2) \exp \left(\frac{a}{\frac{a}{\ln ^{2} \eta_{i, \infty}}\left[\eta_{i, \infty}^{-\left(1+\frac{\xi}{a}\right)} a+\eta_{i, \infty}^{-\left(1+\frac{\xi}{a}\right)} \ln \eta_{i, \infty}\left(\zeta-\sigma_{C}\right)-a+\ln \eta_{i, \infty}\left(\sigma_{C}+a\right)\right]}\right)
$$

The concentration in the bulk is given by:

$$
\Upsilon_{b}(\zeta, \varsigma)=2+(g-2) \exp \left(-\frac{\epsilon}{2} \frac{\varsigma}{\varphi_{b}(\zeta)}\right)
$$

Inserting Eq. (41) in Eq. (46), we obtain:

$$
\Upsilon_{b}(\zeta, \zeta)=2+(g-2) \exp \left(\frac{-\frac{1}{2} \epsilon \zeta}{-\frac{1}{2 \eta_{b}} \zeta^{2}+\frac{1}{\eta_{b}} \zeta+\frac{a}{\ln ^{2} \eta_{i, \infty}}\left[\frac{a-\sigma_{C} \ln \eta_{i, \infty}}{\eta_{i, \infty}}-a+\ln \eta_{i, \infty}\left(\sigma_{C}+a\right)\right]+\varphi_{S}}\right)
$$

which we will use to determine viscosity.

\section{TEMPERATURE-DEPENDENT KINETICS}

The concentration profile will depend on temperature, as the degradation rate will follow Eq. (14). The dimensionless steady state temperature is a function of the distance from the slip interface and the heat flux that arises from this step discontinuity:

$$
\Theta_{d, \infty}(\zeta)=F(\zeta+a)+\Theta_{w} ;-a \leq \zeta \leq 0
$$


$\Theta_{b, \infty}(\zeta)=F a+\Theta_{w} ; 0 \leq \zeta \leq 1$

From these equations, we can see that the temperature does not vary as a function of distance travelled through the channel, only the distance from the wall. Thus:

$k=k(x)$

Reexamining Eq. (27) using the insight from Eq. (49):

$\frac{d c_{B}}{d y}=\frac{k(x)}{v_{y}(x)} c_{A 0}-\frac{k(x)}{2 v_{y}(x)} c_{B}$

and following the method used to develop Eq. (35) yields:

$c_{B}(x, y)=2 c_{A 0}+\left(c_{B 0}-2 c_{A 0}\right) e^{-\frac{k(x)}{2 v_{y}(x)} y}$

The concentration becomes dependent on position in the gap, when temperature dependence is incorporated. Nondimensionalizing Eq. (51) with the dimensionless groups in the Table 2 yields a general solution for concentration in both the bulk:

$\Upsilon(\zeta, \zeta)=2+(g-2) \exp \left[-\frac{r}{2} \exp \left(-\frac{s}{1+\Theta}\right) \frac{\zeta}{\varphi(\zeta)}\right]$

and the drool:

$$
\Upsilon_{d}(\zeta, \zeta)=2+(g-2) \exp \left[-\frac{r}{2} \exp \left(-\frac{s}{1+\Theta_{d}}\right) \frac{\zeta}{\varphi_{d}(\zeta)}\right]
$$

Inserting Eqs. (47) and (40) into Eq. (53), we find:

$$
\Upsilon_{d}(\zeta, \varsigma)=2+(g-2) \exp \left\{-\frac{r}{2} \exp \left(-\frac{s}{1+F(\zeta+a)+\Theta_{w}}\right) \frac{\left.\frac{a}{\ln ^{2} \eta_{i, \infty}}\left[\eta_{i, \infty}^{-\left(1+\frac{5}{a}\right)} a+\eta_{i, \infty}^{-\left(1+\frac{\xi}{a}\right)} \ln \eta_{i, \infty}\left(\zeta-\sigma_{c}\right)-a+\ln \eta_{i, \infty}\left(\sigma_{C}+a\right)\right]\right\}}{(54)}\right.
$$

Eq. (54) yields a drool degradate concentration profile that quickly decreases with distance from the wall, $\zeta$ (see Figure 3). Near the wall, the dimensionless concentration of the degraded polymer is 2 , which indicates that only degradate is present. This observation matches theory since the melt near the wall does not move, and thus spends infinite time degrading. As expected, the concentration of the degraded polymer in the bulk increases as the melt progresses through the extruder (see Figure 3).

The concentration in the bulk layer is given by:

$$
\Upsilon_{b}(\zeta, \zeta)=2+(g-2) \exp \left[-\frac{r}{2} \exp \left(-\frac{s}{1+\Theta_{b}}\right) \frac{\zeta}{\varphi_{b}(\zeta)}\right]
$$

Inserting Eqs. (41) and (48) into Eq. (55), we get:

$$
\Upsilon_{b}(\zeta, \varsigma)=2+(g-2) \exp \left(-\frac{r}{2} \exp \left(-\frac{s}{1+F a+\Theta_{w}}\right) \frac{\varsigma}{-\frac{1}{2 \eta_{b}} \zeta^{2}+\frac{1}{\eta_{b}} \zeta+\frac{a}{\ln ^{2} \eta_{i, \infty}}\left[\frac{a-\sigma_{C} \ln \eta_{i, \infty}}{\eta_{i, \infty}}-a+\ln \eta_{i, \infty}\left(\sigma_{C}+a\right)\right]+\varphi_{S}}\right)
$$

Eq. (56) describes a nearly constant bulk degradate concentration profile across the die cross-section (see Figure 4). The narrow range of $c_{B}$ is unsurprising as the range of velocities is much smaller across the bulk flow. As expected, the concentration of the degraded polymer in the bulk increases as the melt 
progresses through the extruder (see Figure 4). Interestingly, the temperature dependent and independent concentration profiles nearly match.

\section{Flow Rate Estimation}

The volumetric flow rate can be defined by the equation of motion (written in terms of velocity) in Cartesian coordinates (see B.6-2 of [20]):

$$
1=\frac{d}{d \zeta}\left(-\eta\left(M_{\mathrm{w}}, \Theta\right) \frac{d \varphi}{d \zeta}\right)
$$

where the viscosity depends on temperature and molecular weight, both of which depend on position within the flow field. For simplicity, we neglect this temperature dependence. Assuming that the flow is Newtonian, and thus without entanglements, the viscosity can be described using:

$\mu=K M_{\text {w }}$

The weight-average molecular weight is defined by [29]:

$$
M_{\mathrm{w}} \equiv \frac{\sum_{i=1}^{n} x_{i} M_{i}^{2}}{\sum_{i=1}^{n} x_{i} M_{i}}
$$

where $x_{i}$ is the mole fraction of species $i$. Hence, for the binary degradation described by Eq. (9):

$$
M_{\mathrm{w}}=\frac{x_{A} M_{A}{ }^{2}+x_{B} M_{B}{ }^{2}}{x_{A} M_{A}+x_{B} M_{B}}
$$

Using the simplifying assumption that molecular weight of B is exactly half of A yields:

$$
M_{\mathrm{w}}=\frac{x_{A} M_{A}{ }^{2}+\frac{1}{4} x_{B} M_{A}{ }^{2}}{x_{A} M_{A}+\frac{1}{2} x_{B} M_{A}}
$$

Eq. (61) can be rearranged to get:

$$
M_{\mathrm{w}}=\frac{M_{A}\left(x_{A}+\frac{1}{4} x_{B}\right)}{x_{A}+\frac{1}{2} x_{B}}
$$

or, in terms of B:

$$
\frac{M_{\mathrm{w}}}{M_{A}}=\frac{1-\frac{3}{4} x_{B}}{1-\frac{1}{2} x_{B}}
$$

The dimensionless viscosity, $\breve{\eta}$, can be written as:

$$
\breve{\eta} \equiv \frac{\mu}{\mu_{A}}
$$

Replacing both viscosities in Eq. (64) with Eq. (58) and assuming K to be the same for both, because the miscibility of a polymer in a longer chain polymer of itself is high, we find:

$$
\breve{\eta}=\frac{M_{\mathrm{w}}}{M_{A}}
$$


Replacing the molecular weight ratio in Eq. (65) with Eq. (63) yields:

$\breve{\eta}=\frac{1-\frac{3}{4} x_{B}}{1-\frac{1}{2} x_{B}}$

The mole fraction of $B$, written in terms of the concentrations, is:

$x_{B}=\frac{c_{B}}{c_{A}+c_{B}}$

Substituting the stoichiometric definition of $c_{A}$ from Eq. (20) into Eq. (67), we get:

$x_{B}=\frac{c_{B}}{\left(c_{A 0}-\frac{1}{2} c_{B}\right)+c_{B}}$

Simplifying Eq. (68):

$x_{B}=\frac{c_{B}}{c_{A 0}+\frac{1}{2} c_{B}}$

Nondimensionalizing Eq. (69):

$x_{B}=\frac{\Upsilon_{B}}{1+\frac{1}{2} \Upsilon_{B}}$

Substituting Eq. (70) into Eq. (66):

$$
\breve{\eta}=\frac{1-\frac{3}{4}\left(\frac{\Upsilon_{B}}{1+\frac{1}{2} \Upsilon_{B}}\right)}{1-\frac{1}{2}\left(\frac{\Upsilon_{B}}{1+\frac{1}{2} \Upsilon_{B}}\right)}
$$

which simplifies to:

$\breve{\eta}=1-\frac{1}{4} \Upsilon_{B}$

\section{(i) Simplification: Constant Average Concentration Through Taylor Series}

Substituting Eq. (72) into Eq. (57), for any velocity profile $\varphi^{*}(\zeta, \varsigma)$ :

$1=\frac{d}{d \zeta}\left(-\breve{\eta}\left(M_{W}\right) \frac{d \varphi^{*}}{d \zeta}\right)$

The first integration yields:

$\frac{d \varphi^{*}}{d \zeta}=\frac{C_{1}-\zeta}{\left[1-\frac{1}{4} \Upsilon(\zeta, \zeta)\right]}$

To simplify Eq. (74), the drool concentration can be approximated with a Taylor series expansion with respect to $\xi$, and then integrated along the $\varsigma$-axis, and finally divided by the dimensionless length, $l$. This method reduces the concentration expression in Eq. (74) to a constant value, $Y^{*}{ }_{d}$, which does not depend on position. The average drool and bulk concentrations will thus take the form:

$\Upsilon_{d}^{*}=\int_{0}^{1}\left[\frac{\mathrm{T}_{d}(-a)+\mathrm{T}_{d}(0)}{2}\right] d \varsigma$ 


$$
\Upsilon_{b}^{*}=\int_{0}^{1}\left[\frac{\mathrm{T}_{b}(0)+\mathrm{T}_{b}(1)}{2}\right] d \varsigma
$$

Where $\mathrm{T}_{d}$ and $\mathrm{T}_{b}$ represent, for the bulk and drool layers, the lowest order Taylor series about the boundary conditions: the wall $(y=-a)$, the slip interface ( $y=0)$, the midplane of the bulk flow $(y=1)$. Evaluating the truncated Taylor series in Eq. (75) and reinserting them yields:

$$
\Upsilon_{d}^{*}=\frac{1}{2} \int_{0}^{1} 4+(g-2) \exp \left[\frac{-\varsigma r \exp \left(\frac{s}{\Theta_{w}+F a+1}\right)}{-a+\left(\ln \eta_{i, \infty}\right)\left(a+\sigma_{c}\right)+\frac{a}{\eta_{i, \infty}} \frac{\sigma_{c}\left(\ln \eta_{i, \infty}\right)}{\eta_{i, \infty}}}\right] d \varsigma
$$

Integrating Eq. (77) with respect to $\varsigma$ then yields:

$$
\Upsilon_{d}^{*}=\left\{\begin{array}{c}
2 \varsigma-\frac{(g-2)\left(-a+\left(\ln \eta_{i, \infty}\right)\left(a+\sigma_{c}\right)+\frac{a}{\eta_{i, \infty}}-\frac{\sigma_{c}\left(\ln \eta_{i, \infty}\right)}{\eta_{i, \infty}}\right)}{-2 r \exp \left(\frac{s}{\Theta_{w}+F a+1}\right)} \\
\times \exp \left[\frac{-\varsigma r \exp \left(\frac{s}{\Theta_{w}+F a+1}\right)}{-a+\left(\ln \eta_{i, \infty}\right)\left(a+\sigma_{c}\right)+\frac{a}{\eta_{i, \infty}}-\frac{\sigma_{c}\left(\ln \eta_{i, \infty}\right.}{\eta_{i, \infty}}}\right]
\end{array}\right] \mid
$$

or:

$$
\begin{aligned}
\Upsilon_{d}^{*}=2+\frac{(g-2)\left(-a+\left(\ln \eta_{i, \infty}\right)\left(a+\sigma_{c}\right)+\frac{a}{\eta_{i, \infty}}-\frac{\sigma_{c}\left(\ln \eta_{i, \infty}\right)}{\eta_{i, \infty}}\right)}{2 r \exp \left(\frac{s}{\Theta_{w}+F a+1}\right)} \\
\times\left\{\exp \left[\frac{-r \exp \left(\frac{s}{\Theta_{w}+F a+1}\right)}{-a+\left(\ln \eta_{i, \infty}\right)\left(a+\sigma_{c}\right)+\frac{a}{\eta_{i, \infty}}-\frac{\sigma_{c}\left(\ln \eta_{i, \infty}\right)}{\eta_{i, \infty}}}\right]+1\right\}
\end{aligned}
$$

Similarly, for the bulk fluid, the truncated Taylor series can be evaluated around 0 and 1 to approximate the average:

$$
\Upsilon_{b}^{*}=\int_{0}^{1} 2+\frac{(g-2)}{2} \exp \left(\frac{-r \varsigma}{C} \exp \left(\frac{-s}{\Theta_{w}+F a+1}\right)\right)+\frac{(g-2)}{2} \exp \left(\frac{-r \varsigma}{C+\frac{1}{2 \eta_{b}}} \exp \left(\frac{-s}{\Theta_{w}+F a+1}\right)\right) d \varsigma
$$

The integration of Eq. (80) yields: 


$$
\Upsilon_{b}^{*}=2+\frac{C(g-2)}{2 r \exp \left(\frac{s}{\Theta_{w}+F a+1}\right)}\left(\begin{array}{l}
1-\exp \left\{-\frac{r}{C}\left[\exp \left(\frac{s}{\Theta_{W}+F a+1}\right)\right]\right\} \\
+\frac{2 \eta_{b}+\frac{1}{C}}{2 \eta_{b}}\left(1-\exp \left(-\frac{r}{C+\frac{1}{2 \eta_{b}}} \exp \left(\frac{s}{\Theta_{w}+F a+1}\right)\right)\right)
\end{array}\right)
$$

In most circumstances, the average concentration in the bulk fluid is vanishingly low. The average dimensionless concentration of $A$ of the drool fluid calculated in this manner is usually one, as the concentration near the wall is two and the concentration near the slip interface is also vanishingly low.

\section{(ii) Estimating the Drool Flowrate}

Adapting Eq. (74) for the drool layer and assuming a constant average viscosity:

$$
\frac{d \varphi^{*}{ }_{d}}{d \zeta}=\frac{C_{1}-\zeta}{\left[1-\frac{1}{4} \Upsilon^{*}{ }_{b}\right]}
$$

The boundary conditions for the drool layer are the wall, where the drool velocity is zero, and the drool slip boundary, where the shear stress is equal to the cohesive fracture strength. Applying the first boundary condition, the slip shear stress can be expressed as a function of the cohesive fracture strength of the polymer, $\sigma_{c}$, and the viscosity at the interface, $\eta_{i, \infty}$ :

$$
\left.\frac{d \varphi_{d}^{*}}{d \zeta}\right|_{\xi=0}=\frac{\sigma_{C}}{\eta_{i, \infty}}=\frac{C_{1}-0}{\left[1-\frac{1}{4} \Upsilon^{*}{ }_{d}\right]}
$$

and thus:

$$
C_{1}=\frac{\sigma_{c}\left(1-\frac{1}{4} \Upsilon_{d}^{*}\right)}{\eta_{i, \infty}}
$$

Inserting Eq. (84) into (82) and integrating yields:

$$
\varphi_{d}^{*}=\frac{-1}{2\left(1-\frac{1}{4} \Upsilon_{d}^{*}\right)} \zeta^{2}+\frac{\sigma_{c}}{\eta_{i, \infty}} \zeta+C_{2}
$$

Applying the second boundary condition, where the velocity drops to zero at the wall:

$$
\varphi_{d,-a}^{*}=0=\frac{-(-a)^{2}}{2\left(1-\frac{1}{4} \Upsilon_{d}^{*}\right)}+\frac{\sigma_{c}}{\eta_{i, \infty}}(-a)+C_{2}
$$

and:

$$
C_{2}=\frac{a \sigma_{c}}{\eta_{i, \infty}}+\frac{a^{2}}{2\left(1-\frac{1}{4} \Upsilon_{d}^{*}\right)}
$$

Substituting into Eq. (85) yields: 
$\varphi_{d}^{*}=\frac{-1}{2\left(1-\frac{1}{4} \Upsilon^{*}{ }_{d}\right)} \zeta^{2}-\frac{\sigma_{c}}{\eta_{i, \infty}} \zeta+a\left(\frac{\sigma_{c}}{\eta_{i, \infty}}+\frac{a}{2\left(1-\frac{1}{4} \Upsilon_{d}^{*}\right)}\right)$

Figure 5 shows the drool velocity profile as nearly linear (see similar shape in [16]). The velocity of the bulk is higher than the drool, despite the lower drool viscosity brought about by the degraded polymer. The drool flowrate can be derived from the velocity profile, Eq. (88), by integrating along the cross-sectional area and then multiplying by 2 to account for the symmetry of the die drool flow:

$Q_{d}^{*}=2 \int_{0}^{W} \int_{-a}^{0} \varphi_{d}^{*} d \zeta d W^{*}$

Substituting Eq. (88) into Eq. (89):

$Q_{d}^{*}=2 \int_{0}^{W} \int_{-a}^{0}\left[\frac{-1}{2\left(1-\frac{1}{4} \Upsilon^{*}{ }_{d}\right)} \zeta^{2}+\frac{\sigma_{c}}{\eta_{i, \infty}} \zeta+a\left(\frac{\sigma_{c}}{\eta_{i, \infty}}+\frac{a}{2\left(1-\frac{1}{4} \Upsilon_{d}^{*}\right)}\right)\right] d \zeta d W^{*}$

Integrating twice yields:

$Q_{d}^{*}=2 W \int_{-a}^{0}\left[\frac{-1}{2\left(1-\frac{1}{4} \Upsilon_{d}^{*}\right)} \zeta^{2}+\frac{\sigma_{c}}{\eta_{i, \infty}} \zeta+a\left(\frac{\sigma_{c}}{\eta_{i, \infty}}+\frac{a}{2\left(1-\frac{1}{4} \Upsilon_{d}^{*}\right)}\right)\right] d \zeta$

and applying the boundary conditions gives:

$Q_{d}^{*}=\left.2 W\left[\frac{-1}{6\left(1-\frac{1}{4} \Upsilon_{d}^{*}\right)} \zeta^{3}+\frac{\sigma_{c}}{2 \eta_{i, \infty}} \zeta^{2}+a\left(\frac{\sigma_{c}}{\eta_{i, \infty}}+\frac{a}{2\left(1-\frac{1}{4} \Upsilon_{d}^{*}\right)}\right) \zeta\right]\right|_{\zeta=-a} ^{\zeta=0}$

$Q_{d}^{*}=\frac{2 W a^{2}}{\left(1-\frac{1}{4} \Upsilon{ }^{*}\right)}\left[\frac{\sigma_{c}\left(1-\frac{1}{4} \Upsilon^{*}{ }_{d}\right)}{2 \eta_{i, \infty}}+\frac{a}{3}\right]$

\section{(iii) Estimating the Bulk Flow Rate}

Adapting Eq. (74) for the bulk melt:

$1=\frac{d}{d \zeta}\left(-\eta *\left(M_{W}\right) \frac{d \varphi_{b, 2}}{d \zeta}\right)$

and integrating gives:

$\zeta=-\imath *\left(M_{W}\right) \frac{d \varphi_{b}^{*}}{d \zeta} \quad C_{1}$

Assuming that the viscosity can be calculated from an average:

$\zeta=-\left[1-\frac{1}{4} \Upsilon_{b} t(\zeta, \zeta)\right] \frac{d \varphi_{b}^{*}}{d \zeta} \quad C_{1}$

At $\zeta=1$, the dimensionless velocity peaks, thus:

$C_{1}=1$

Inserting Eq. (97) into Eq. (96)

$\zeta=-\left[1-\frac{1}{4} \Upsilon_{b}+(\zeta, \zeta)\right] \frac{d \varphi^{*}{ }_{b}}{d \zeta} 1$ 
Solving for the velocity profile:

$$
\frac{d \varphi_{b}^{*}}{d \zeta}=\frac{1-\zeta}{1-\frac{1}{4} \Upsilon_{b}(\zeta, \zeta)}
$$

Following the procedure that we used above for the drool layer, we average the concentration profile over the crossflow direction:

$$
\frac{d \varphi^{*}{ }_{b}}{d \zeta}=\frac{1-\zeta}{1-\frac{1}{4} \Upsilon^{*}}
$$

Integrating Eq. (100) with respect to $\zeta$ :

$\varphi_{b}^{*}=\frac{-1}{2\left(1-\frac{1}{4} \Upsilon_{b}^{*}\right)} \zeta^{2}+\frac{1}{1-\frac{1}{4} \Upsilon_{b}^{*}} \zeta+C_{1}$

At the slip interface, the bulk velocity is defined as the drool velocity at the slip layer added to the slip velocity [the slip velocity can be calculated from Eq.(88)]:

$\left.\varphi_{b}^{*}\right|_{\zeta=0}=\left.\varphi_{d}^{*}\right|_{\zeta=0}+\varphi_{s}$

Substituting Eq. (102) into Eq. (101) at the boundary reveals:

$C_{1}=\left.\varphi^{*}\right|_{\zeta=0}+\varphi_{s}$

and substituting the bulk velocity at the boundary into Eq. (103) gives:

$C_{1}=a\left(\frac{\sigma_{c}}{\eta_{i, \infty}}+\frac{a}{2\left(1-\frac{1}{4} \Upsilon_{d}^{*}\right)}\right)+\varphi_{s}$

Substituting Eq. (104) into (101), we get:

$\varphi_{b}^{*}=\frac{-1^{2}}{2\left(1-\frac{1}{4} \Upsilon^{*}{ }_{b}\right)} \zeta^{2}+\frac{1}{1-\frac{1}{4} \Upsilon_{b}^{*}} \zeta+a\left(\frac{\sigma_{c}}{\eta_{i, \infty}}+\frac{a}{2\left(1-\frac{1}{4} \Upsilon_{d}^{*}\right)}\right)+\varphi_{s}$

As expected, the bulk velocity profile is curved (see Figure 6). The velocity profile expressed by Eq. (105) resembles the profile found in Giacomin and Gilbert [16], but the velocity at the slip interface is greater than that found in [16]. The bulk flowrate can be derived from the velocity profile by integrating along the crosssectional area (above the midplane) and by then multiplying by 2 (to account for the flow symmetry):

$Q_{b}^{*}=2 \int_{0}^{W} \int_{0} \varphi_{b}^{*} d \zeta d W^{*}$

Substituting Eq. (105) into Eq. (106) gives:

$Q_{b}^{*}=2 \int_{0}^{W} \int_{0}^{1}\left(\frac{-1^{2}}{2\left(1-\frac{1}{4} \Upsilon_{b}^{*}\right)} \zeta^{2}+\frac{1}{1-\frac{1}{4} \Upsilon_{b}^{*}} \zeta+a\left(\frac{\sigma_{c}}{\eta_{i, \infty}}+\frac{a}{2\left(1-\frac{1}{4} \Upsilon_{d}^{*}\right)}\right)+\varphi_{s}\right) d \zeta d W^{*}$

and then integrating:

$Q_{b}^{*}=2 W\left(\frac{-1^{2}}{6\left(1-\frac{1}{4} \Upsilon_{b}^{*}\right)} \zeta^{3}+\frac{1}{2\left(1-\frac{1}{4} \Upsilon_{b}^{*}\right)} \zeta^{2}+\left.\left[a\left(\frac{\sigma_{c}}{\eta_{i, \infty}}+\frac{a}{2\left(1-\frac{1}{4} \Upsilon_{d}^{*}\right)}\right)+\varphi_{s}\right] \zeta\right|_{\zeta=0} ^{\zeta=1}\right.$

Applying the boundary conditions to the definite integral: 
$Q_{b}^{*}=\frac{2 W}{\left(1-\frac{1}{4} \Upsilon_{b}^{*}\right)}\left\{\frac{1}{3}+\left(1-\frac{1}{4} \Upsilon_{b}^{*}\right)\left[a\left(\frac{\sigma_{c}}{\eta_{i, \infty}}+\frac{a}{2\left(1-\frac{1}{4} \Upsilon_{d}^{*}\right)}\right)+\varphi_{s}\right]\right\}$

Following Gander and Giacomin [10], we normalize the drool flow rate with bulk flow rate to obtain the build up ratio [15]:

$$
B R \equiv \frac{Q_{d}^{*}}{Q_{b}^{*}}
$$

Analytically, this ratio can be obtained from Eqs. (93) and (109):

$$
B R \equiv \frac{a^{2}\left(1-\frac{1}{4} \Upsilon_{b}^{*}\right)\left[\frac{\sigma_{c}\left(1-\frac{1}{4} \Upsilon^{*}{ }_{d}\right)}{2 \eta_{i, \infty}}+\frac{a}{3}\right]}{\left(1-\frac{1}{4} \Upsilon_{d}^{*}\right)\left\{\frac{1}{3}+\left(1-\frac{1}{4} \Upsilon_{b}^{*}\right)\left[a\left(\frac{\sigma_{c}}{\eta_{i, \infty}}+\frac{a}{2\left(1-\frac{1}{4} \Upsilon_{d}^{*}\right)}\right)+\varphi_{s}\right]\right\}}
$$

The buildup ratio described by Eq. (111) reveals a larger buildup ratio (by about $20 \%$ ) compared to the work done by Giacomin and Gilbert [16]. This is because the lower drool viscosity enhances the drool flow rate.

\section{(iv) Reworked Example: Sheet Extrusion Drool Rate}

A plastic sheet manufacturer aims to estimate the steady drool rate for molten nylon, to be considered for her extrusion operations. For her process, she knows that $T_{w}=T_{0}=423 \mathrm{~K}, \tau_{c}=0.18 \mathrm{MPa}, v_{s}=30 \mathrm{~mm} / \mathrm{s}, k=0.1 \mathrm{~W} / \mathrm{mK}, C_{p}=2.0 \mathrm{~J} / \mathrm{GK}$, $\rho=0.90 \mathrm{~g} / \mathrm{mL}, \mu_{w}=600 \mathrm{~Pa}-\mathrm{s}, L=0.50 \mathrm{~m}, \Delta P=-31 \mathrm{MPa}, c_{B 0}=0, c_{A 0}=1.0 \mathrm{M}$ and $w=1.0 \mathrm{~m}$. From Ref. [30], for nylon 6,6 she gleans $A=20 \mathrm{~s}^{-1}$ and $E_{a}=129 \mathrm{kJmol}^{-1}$. She first calculates the inlet resin purity index from Eq. (38):

$g=\frac{c_{B 0}}{c_{A 0}}=0$

and then determines the drool layer thickness using Eq. (18) of [15]:

$d=\frac{h}{2}+\frac{\tau_{c} L}{\Delta P}=0.097 \mathrm{~mm}$

The bulk fluid thickness is derived from the defining relation for $d$ :

$b=\frac{h}{2}-d=2.9 \mathrm{~mm}$

Applying these values to Eq. (5), and using the cohesive fracture strength, she finds:

$q_{i}=\mathrm{v}_{s} \tau_{c}=5.4 \mathrm{~kW} / \mathrm{m}^{2}$

This heat flux can be nondimensionalized as: 
$F=\frac{q_{i} \mathrm{~b}}{k T_{0}}=0.348$

She then nondimensionalizes the drool thickness:

$a=\frac{d}{b}=0.033$

and width

$W=\frac{w}{b}=344$

Using the values above, the temperature profiles can be described with Eqs. (47) and (48). The dimensionless temperature at the wall is zero because, in this example, it matches the initial temperature:

$\Theta_{d, \infty}=0.348(\zeta+0.033) ;-a \leq \zeta \leq 0$

$\Theta_{b, \infty}=\Theta_{i, \infty}=1.16 \times 10^{-2} ; 0 \leq \zeta \leq 1$

Using the method of Gilbert and Giacomin [16], she calculates the interface viscosity:

$\eta_{i, \infty}=\eta_{b}=\exp \left(-\Theta_{i, \infty}\right)=0.88$

Before estimating the flow rate, she finds the dimensionless fracture strength, dimensionless coefficients and slip velocity:

$$
\begin{aligned}
& \sigma_{c}=\frac{-\tau_{c} L}{b \Delta P}=1 \\
& \varphi_{s}=\frac{v_{s} \mu_{w} L}{b^{2} \Delta P}=0.034 \\
& s=\frac{E_{a}}{R T_{0}}=36.7 \\
& r=\frac{A \mu_{W} L^{2}}{b^{2} \Delta P}=13.6
\end{aligned}
$$

Substituting Eqs. (121) through (125) into Eq. (54), she finds

$$
\begin{aligned}
& \Upsilon_{d}=2-2 \exp \left\{\frac{3.37 \varsigma \exp \left[\frac{-36.7}{0.348 \zeta+1.01}\right]}{\frac{0.012(\zeta-1)}{0.88^{30.3 \xi+1}}-\frac{0.033}{0.88^{30.3 \xi+1}}+0.17}\right\} ;-a \leq \zeta \leq 0 \\
& \quad=2-2 \exp \left\{\frac{-1.18 \times 10^{-15} \varsigma}{-0.57 \zeta^{2}+1.13 \zeta+0.238}\right\} ; 0 \leq \zeta \leq 1
\end{aligned}
$$


and then prepares Figure 3 and Figure 4. Using the Taylor series approximations [Eqs. (79) and (81)], she finds the average dimensionless concentration in the bulk and drool layers:

$\Upsilon_{d}^{*}=1 ;-a \leq \zeta \leq 0$

$\Upsilon_{b}^{*}=7.2 \times 10^{-16} \approx 0 ; 0 \leq \zeta \leq 1$

Using these average degraded polymer concentrations, she describes the velocity profile using Eqs. (88) and (105) and then prepares Figure 5 and Figure 6:

$\varphi_{b}^{*}=-0.5 \zeta^{2}+\zeta+0.383 ; 0 \leq \zeta \leq 1$

$\varphi_{d}^{*}=-0.5 z^{2}-1.01 z+0.038 ;-a \leq \zeta \leq 0$

Using the dimensionless values above, she calculates the expected flow rates from Eqs. (93) and (109):

$Q_{d}^{*}=\frac{2 W a^{2}}{\left(1-\frac{1}{4} \Upsilon^{*}{ }_{d}\right)}\left[\frac{\sigma_{c}\left(1-\frac{1}{4} \Upsilon^{*}{ }_{d}\right)}{2 \eta_{i, \infty}}+\frac{a}{3}\right]=0.437$

$Q_{b}^{*}=\frac{2 W}{\left(1-\frac{1}{4} \Upsilon_{b}^{*}\right)}\left\{\frac{1}{3}+\left(1-\frac{1}{4} \Upsilon_{b}^{*}\right)\left[a\left(\frac{\sigma_{c}}{\eta_{i, \infty}}+\frac{a}{2\left(1-\frac{1}{4} \Upsilon_{d}^{*}\right)}\right)+\varphi_{s}\right]\right\}=490$

Taking the ratio of Eq. (132) and (133), she finally obtains the buildup ratio:

$B R=\frac{Q_{d}^{*}}{Q_{b}^{*}}=9.0 * 0^{-4}$

Mindful of the erratum to our citation [16], she finds that this is about 20\% higher than the buildup ratio calculated for the same example (sans polymer degradation) in Question 1 of [16]. Otherwise put, in this worked example, polymer scission significantly accelerates drool.

\section{VALIDATING THE NEGLECT OF DIFFUSION}

Whereas Eq. (15) includes diffusion, our equation for time on-stream, Eq. (22), neglects diffusion. In this section, we explore this assumption by first identifying the relevant dimensionless groups and then performing an order of magnitude analysis to compare convection with axial and transverse diffusion.

\section{a. Defining Relevant Dimensionless Groups}

We begin with the equation of continuity for species $\alpha$ (Eq. 19.1-10 of [20]):

$\frac{\partial c_{\alpha}}{\partial t}=-\left(\nabla \cdot \mathrm{N}_{\alpha}\right)+R_{\alpha}, \alpha=A, B$

which is a form of Eq. (15). Substituting Eq. (V) from Table 17.8-1 of [20] into Eq. (135), we find:

$\frac{\partial c_{\alpha}}{\partial t}=-\left(\nabla \cdot c_{\alpha} \boldsymbol{v}^{*}\right)-\left(\nabla \cdot \boldsymbol{J}_{\alpha}^{*}\right)+R_{\alpha}$

which describes the accumulation of species $\alpha$. From left to right, the terms on the right side of the Eq. (136) describe convective mass transfer, diffusive mass 
transfer and species generation/consumption through chemical reaction. The left side of (136) vanishes at steady state, and we replace the reaction term with our result for first order kinetics, Eq. (13), to get:

$0=-\left(\nabla \cdot c_{B} v^{*}\right)-\left(\nabla \cdot J_{B}^{*}\right)+k c_{A}$

Inserting Fick's law of diffusion:

$\boldsymbol{J}_{\boldsymbol{B}}^{*}=-c D_{B A} \nabla x_{B}$

into Eq. (137) and expanding the divergence for Cartesian coordinates (defined in Figure 2), we find:

$0=-\left(\begin{array}{l}\frac{\partial\left(c_{B} v_{x}\right)}{\partial x}+\frac{\partial\left(c_{B} v_{y}\right)}{\partial y}+\frac{\partial\left(c_{B} v_{z}\right)}{\partial z} \\ +\mathscr{O}_{B A}\left(\frac{\partial\left(c \frac{\partial x_{B}}{\partial x}\right)}{\partial x}+\frac{\partial\left(c \frac{\partial x_{B}}{\partial y}\right)}{\partial y}+\frac{\partial\left(c \frac{\partial x_{B}}{\partial z}\right)}{\partial z}\right)\end{array}\right)+k c_{A}$

We next simplify Eq. (139) for uniaxial $y$ direction flow by setting $v_{x}=v_{z}=0$, and after applying die $z$-coordinate symmetry to neglect concentration differences in the $z$ direction (zero lateral diffusion), we get:

$0=-\frac{\partial\left(c_{B} v_{y}\right)}{\partial y}-\mathscr{D}_{B A}\left(\frac{\partial\left(c \frac{\partial x_{B}}{\partial x}\right)}{\partial x}+\frac{\partial\left(c \frac{\partial x_{B}}{\partial y}\right)}{\partial y}\right)+k\left(c_{A 0}-\frac{1}{2} c_{B}\right)$

where:

$c_{A}=c_{A 0}-\frac{1}{2} c_{B}$

and:

$c_{B}=x_{B} C$

We make Eq. (140) dimensionless using the expressions in Table 2 to get:

$0=-\varphi \frac{b^{2} \Delta P}{\mu L}\left(\frac{\partial\left(\Upsilon \cdot c_{\mathrm{A} 0}\right)}{\partial(\varsigma \cdot L)}\right)-\mathscr{O D}_{B A}\left(\frac{\partial^{2}\left(\Upsilon \cdot \mathrm{c}_{\mathrm{A} 0}\right)}{\partial(\zeta \cdot b)^{2}}+\frac{\partial^{2}\left(\Upsilon \cdot \mathrm{c}_{\mathrm{A} 0}\right)}{\partial(\zeta \cdot L)^{2}}\right)+k\left(c_{A 0}-\frac{1}{2} \Upsilon_{A 0}\right)$

Simplifying Eq. (143) and using the definition of dimensionless velocity yields:

$0=-\frac{v}{L k} \frac{\partial \Upsilon}{\partial \varsigma}-\frac{\vartheta_{B A}}{k b^{2}} \frac{\partial^{2} \Upsilon}{\partial \zeta^{2}}-\frac{\partial_{B A}}{k L^{2}} \frac{\partial^{2} \Upsilon}{\partial \varsigma^{2}}+\left(1-\frac{1}{2} \Upsilon\right)$

Transverse and axial species diffusion is described by the second and third terms in Eq. (144). Rearranging Eq. (144) as:

$0=-\frac{\mathscr{O}_{B A}}{k L^{2}}\left[\frac{L v}{\mathscr{O}_{B A}} \frac{\partial \Upsilon}{\partial \varsigma}+\left(\left(\frac{L}{b}\right)^{2} \frac{\partial^{2} \Upsilon}{\partial \zeta^{2}}+\frac{\partial^{2} \Upsilon}{\partial \varsigma^{2}}\right)\right]+\left(1-\frac{1}{2} \Upsilon\right)$

we uncover three dimensionless groups: the second Damköhler number $\left(\mathrm{Da}_{\mathrm{II}}\right)$, the mass-transfer Péclet number ( Pé $_{\mathrm{m}}$ ) and the aspect ratio. We therefore determine a necessary condition for neglecting transverse diffusion: 
$\left(\frac{L}{b}\right)^{2} \gg 1$

which when satisfied, Eq. (145) simplifies to:

$0=-\frac{1}{\mathrm{Da}_{\mathrm{II}}}\left[\frac{L v}{\partial_{B A}} \frac{\partial \Upsilon}{\partial \zeta}+\left(\frac{L}{b}\right)^{2} \frac{\partial^{2} \Upsilon}{\partial \zeta^{2}}\right]+\left(1-\frac{1}{2} \Upsilon\right)$

Similarly, the axial diffusion term can be neglected if:

Pé ${ }_{\mathrm{m}} \equiv \frac{L v}{V_{B A}} \gg\left(\frac{L}{b}\right)^{2}$

which when satisfied, Eq. (147) simplifies to:

$0=-\frac{1}{\mathrm{Da}_{\mathrm{II}}}\left[\operatorname{Pé}_{\mathrm{m}} \frac{\partial \Upsilon}{\partial \varsigma}\right]+\left(1-\frac{1}{2} \Upsilon\right)$

For most industrial extrusion operations, the necessary conditions, Eqs. (146) and (148), are satisfied, which justifies the assumptions upon which our solution depend.

\section{b. Order of Magnitude Analysis: Axial Diffusion}

We now further support Eqs. (146) and (148) by performing an order of magnitude analysis (see, for instance, EXAMPLE 1.3-3 of [31]) to compare bulk convection with axial and transverse diffusion.

Eq. (146) can be written as:

$\left(\frac{L}{b}\right)^{2} \frac{\partial^{2} \Upsilon}{\partial \zeta^{2}} \gg \frac{\partial^{2} \Upsilon}{\partial \varsigma^{2}}$

which compares the axial and transverse diffusion terms. Expanding the

derivatives in Eq. (150), we get:

$\left(\frac{L}{b}\right)^{2} \frac{\partial}{\partial \zeta}\left(\frac{\partial \Upsilon}{\partial \zeta}\right) \gg \frac{\partial}{\partial \varsigma}\left(\frac{\partial \Upsilon}{\partial \varsigma}\right)$

To determine the order of magnitude, we replace each partial differential with a total difference between the maximum and minimum limits of each variable:

$\frac{L^{2}}{b^{2}} \frac{1}{\Delta \zeta}\left(\frac{\Delta \Upsilon}{\Delta \zeta}\right) \gg \frac{1}{\Delta \varsigma}\left(\frac{\Delta \Upsilon}{\Delta \varsigma}\right)$

where these total differences are:

$\Delta \Upsilon \sim O\left(Y_{L}-\Upsilon_{0}\right) \sim O(2-0)$

$\Delta \varsigma \sim O\left(\varsigma_{L}-\varsigma_{0}\right) \sim O(1-0)$

$\Delta \zeta \sim O\left(\zeta_{b}-\zeta_{d}\right) \sim O\left[1-\left(-\frac{d}{b}\right)\right]$

which, when substituted into Eq. (152), gives:

$\left(\frac{L}{b}\right)^{2} \frac{1}{1+d / b}\left(\frac{2}{1+d / b}\right) \gg \frac{1}{1}\left(\frac{2}{1}\right)$

which simplifies to: 


$$
\left(\frac{L}{h}\right)^{2} \gg 1
$$

As long as this condition is satisfied, as it normally is in plastics extrusion, the axial diffusion can be neglected. We next perform a similar analysis to explore transverse diffusion.

\section{c. Order of Magnitude Analysis: Transverse Diffusion}

We begin by comparing axial convection and transverse diffusion:

$$
\frac{v L}{D_{B A}} \frac{\partial \Upsilon}{\partial \zeta} \gg\left(\frac{L}{b}\right)^{2} \frac{\partial^{2} \Upsilon}{\partial \zeta^{2}}
$$

Replacing the derivatives with their total differences, we obtain:

$$
\frac{v L}{D_{B A}} \frac{\Delta \Upsilon}{\Delta \zeta} \gg\left(\frac{L}{b}\right)^{2} \frac{1}{\Delta \zeta}\left(\frac{\Delta \Upsilon}{\Delta \zeta}\right)
$$

Substituting Eqs. (153) through (155) into Eq. (159), we find:

$$
\frac{v L}{D_{B A}} \frac{2}{1} \gg\left(\frac{L}{b}\right)^{2} \frac{1}{1+\frac{d}{b}}\left(\frac{2}{1+\frac{d}{b}}\right)
$$

which simplifies to:

$$
\mathrm{Pé}_{\mathrm{m}} \equiv \frac{v L}{D_{B A}} \gg\left(\frac{L}{h}\right)^{2}
$$

the necessary condition for neglecting transverse diffusion. As long as this condition is satisfied, as it normally is in plastics extrusion, the transverse diffusion can be neglected.

\section{CONCLUSION}

We have chosen the simplest relevant model to explore the influence of polymer degradation on drool rate: polymer scission by an even split with first order kinetics. We combine this will with our previously examined model for cohesive slip with both slip heating and viscous dissipation. We find that scission can, indeed, accelerate drool. We include one worked example to show the usefulness of our work. Finally, we perform an order of magnitude analysis to arrive at necessary conditions for neglecting axial and transverse diffusion, foundations upon which our results solidly rest.

\section{ACKNOWLEDGMENT}

G. Hoy is grateful for the financial support of the Province of Alberta through the Rutherford Scholarship, and to Queen's University for the Principal's Scholarship. P.H. Gilbert is grateful for an International Tuition Award from the School of Graduate Studies of Queen's University at Kingston. A.J. Giacomin is indebted to the Faculty of Applied Science and Engineering of Queen's University at Kingston, for its support through a Research Initiation Grant (RIG). This research was undertaken, in part, thanks to funding from the Canada Research Chairs program of the Government of Canada for the Natural Sciences and 
Engineering Research Council of Canada (NSERC) Tier 1 Canada Research Chair in Rheology.

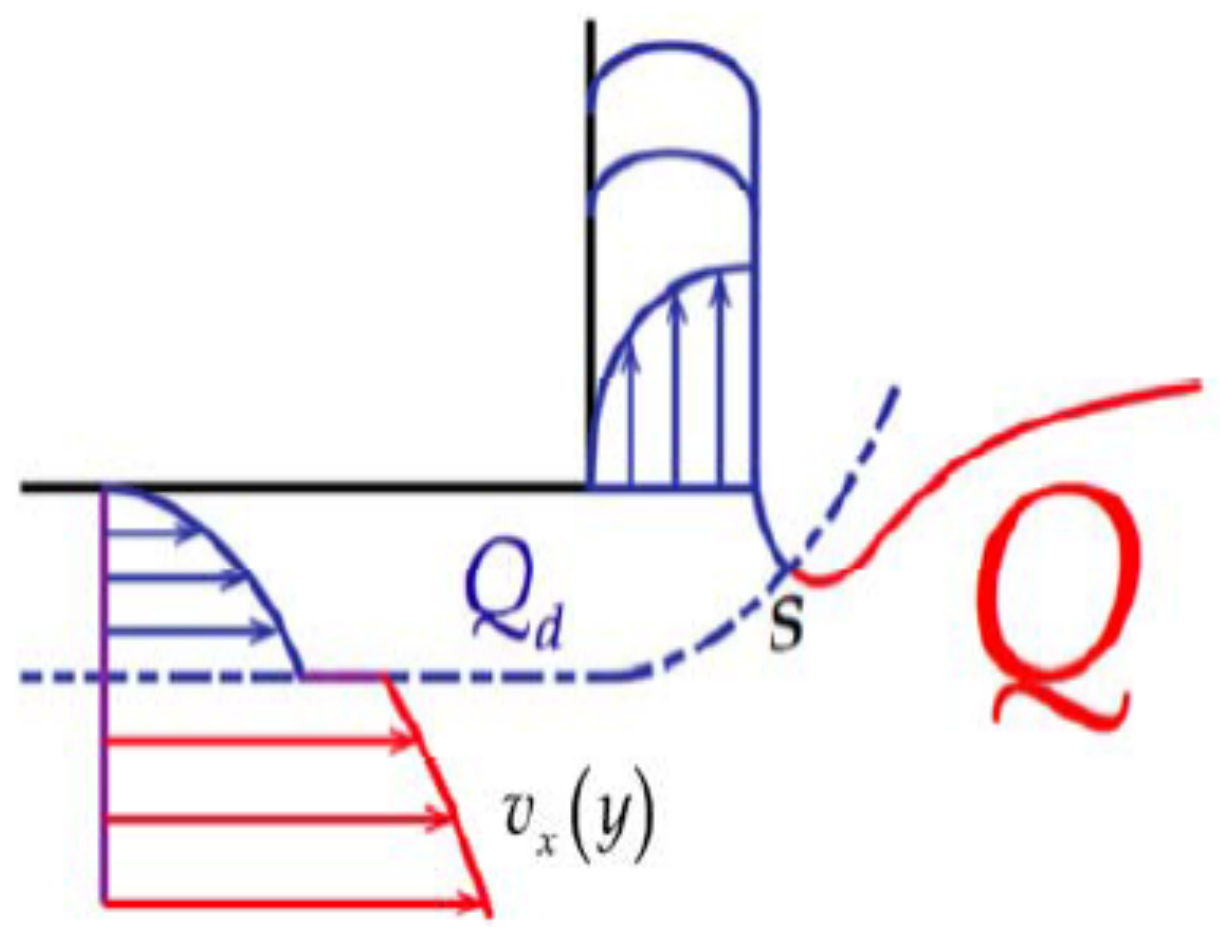

Figure 1: Flow profile for proposed cohesive slip creation of die drool [15] 


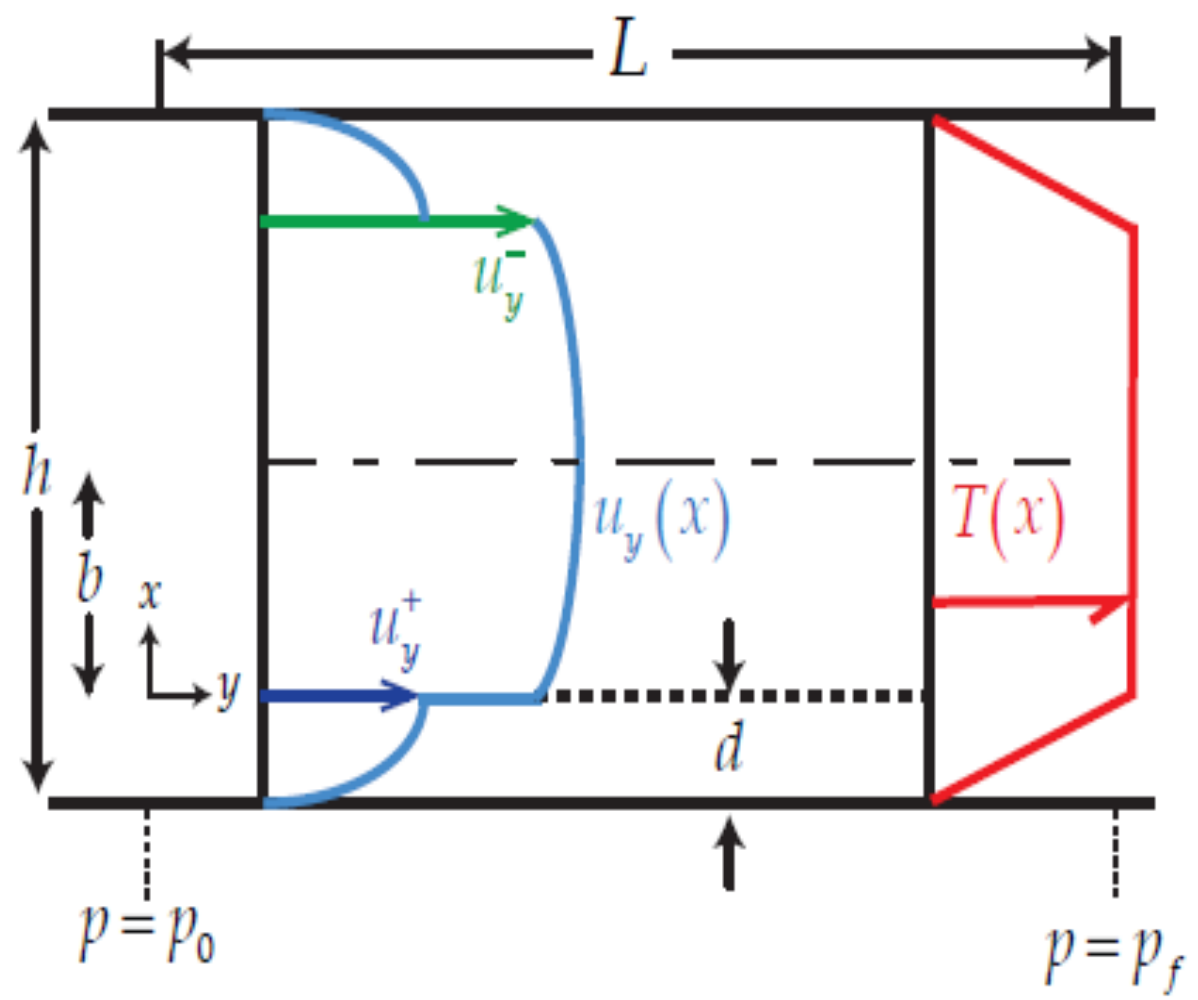

Figure 2: Velocity profiles and concomitant steady state temperature profiles before adjustment for concentration effects on viscosity [15] 


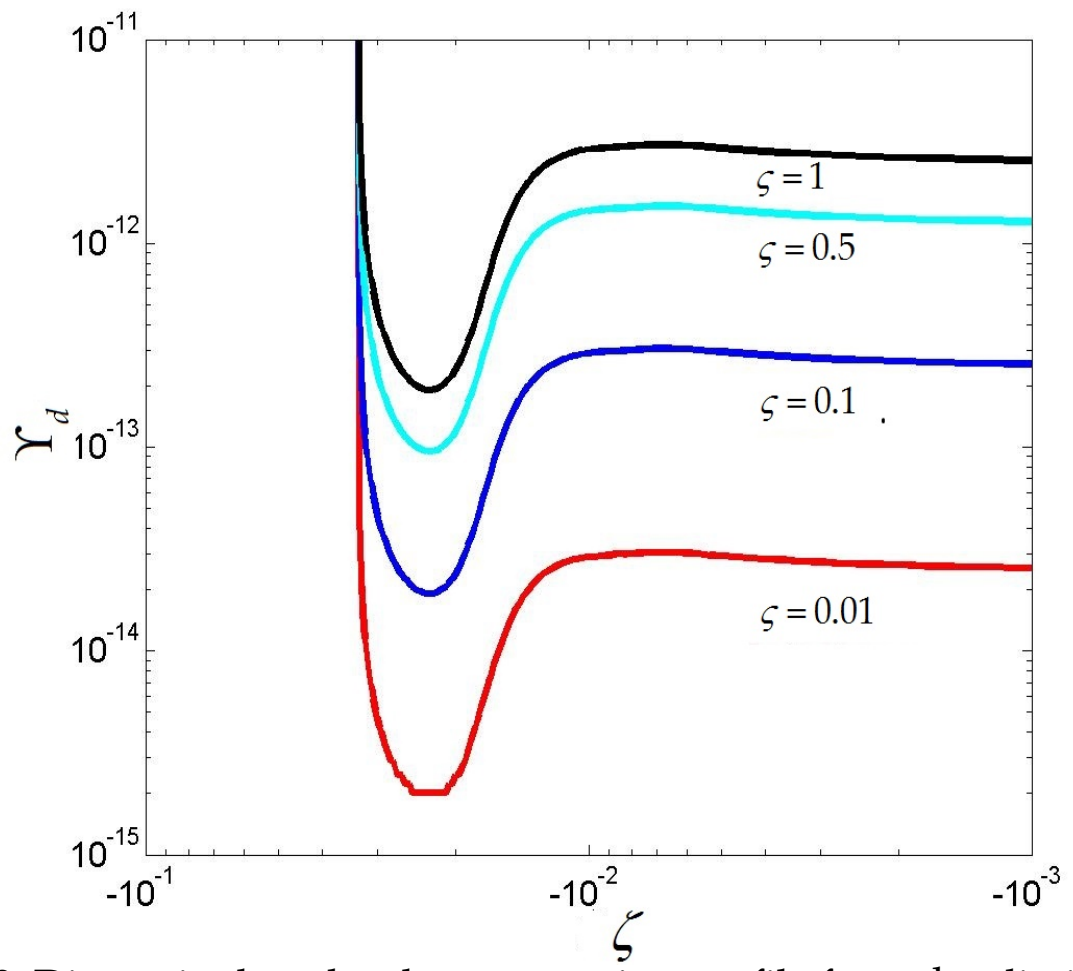

Figure 3: Dimensionless drool concentration profile from the slip interface to the wall 


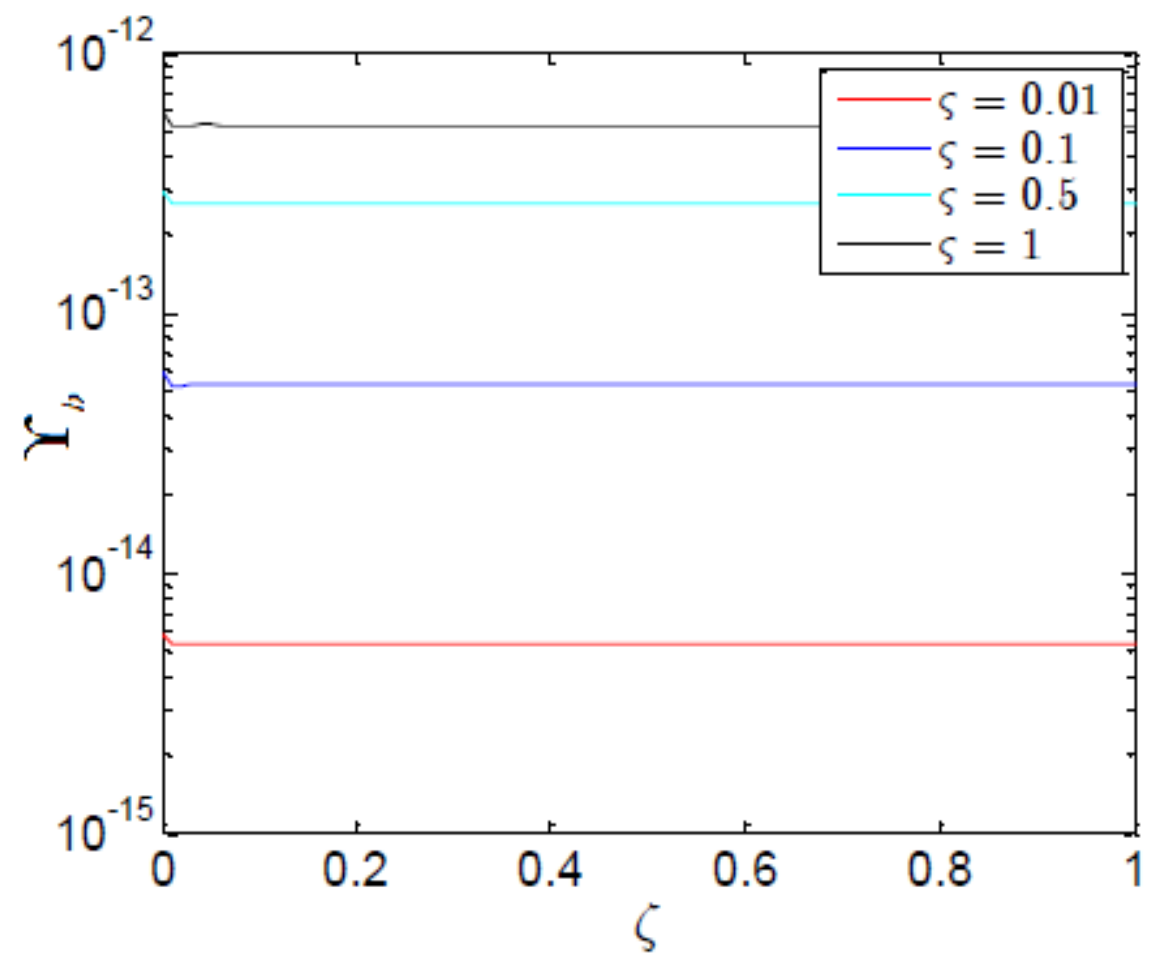

Figure 4: Dimensionless bulk concentration profile from the slip interface to the midplane of the bulk flow 


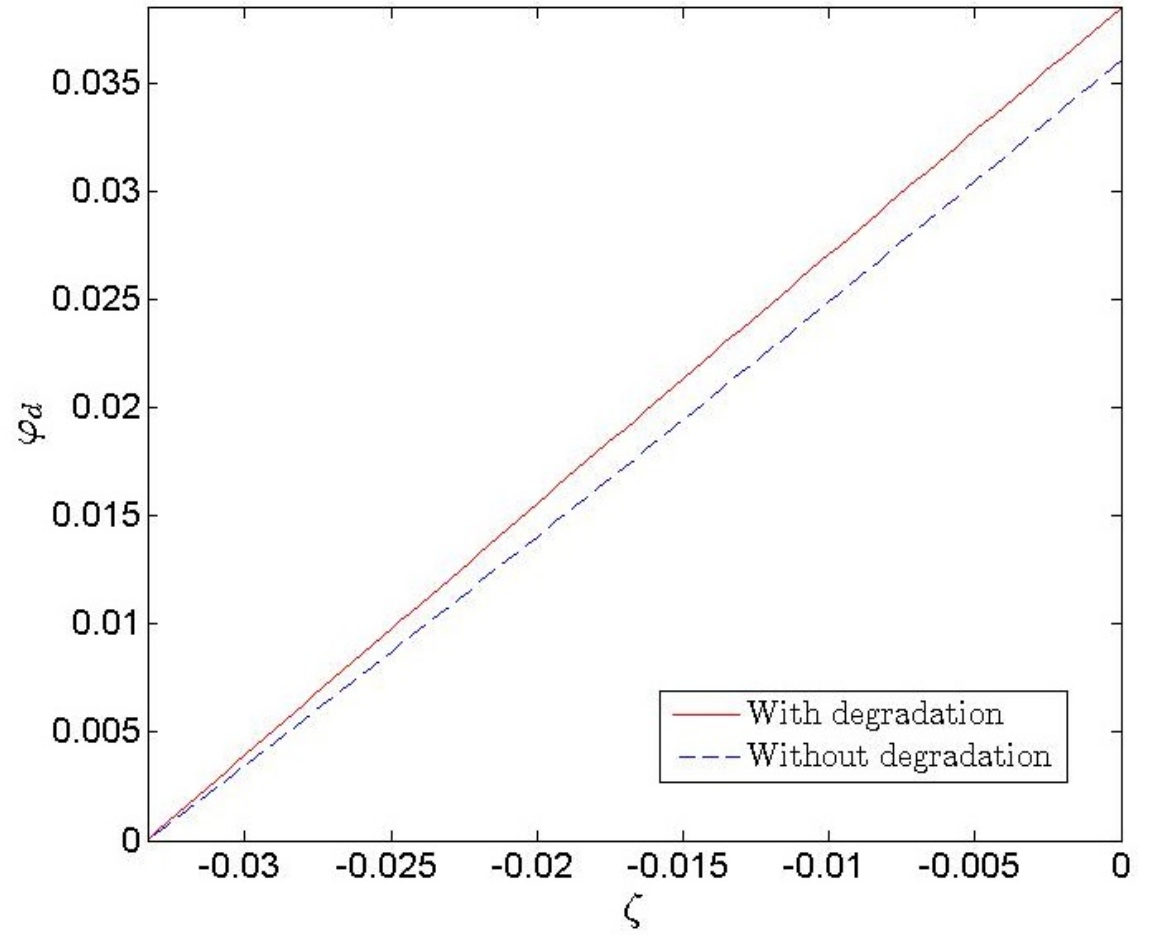

Figure 5: Dimensionless drool velocity profiles from the wall to the slip interface. 


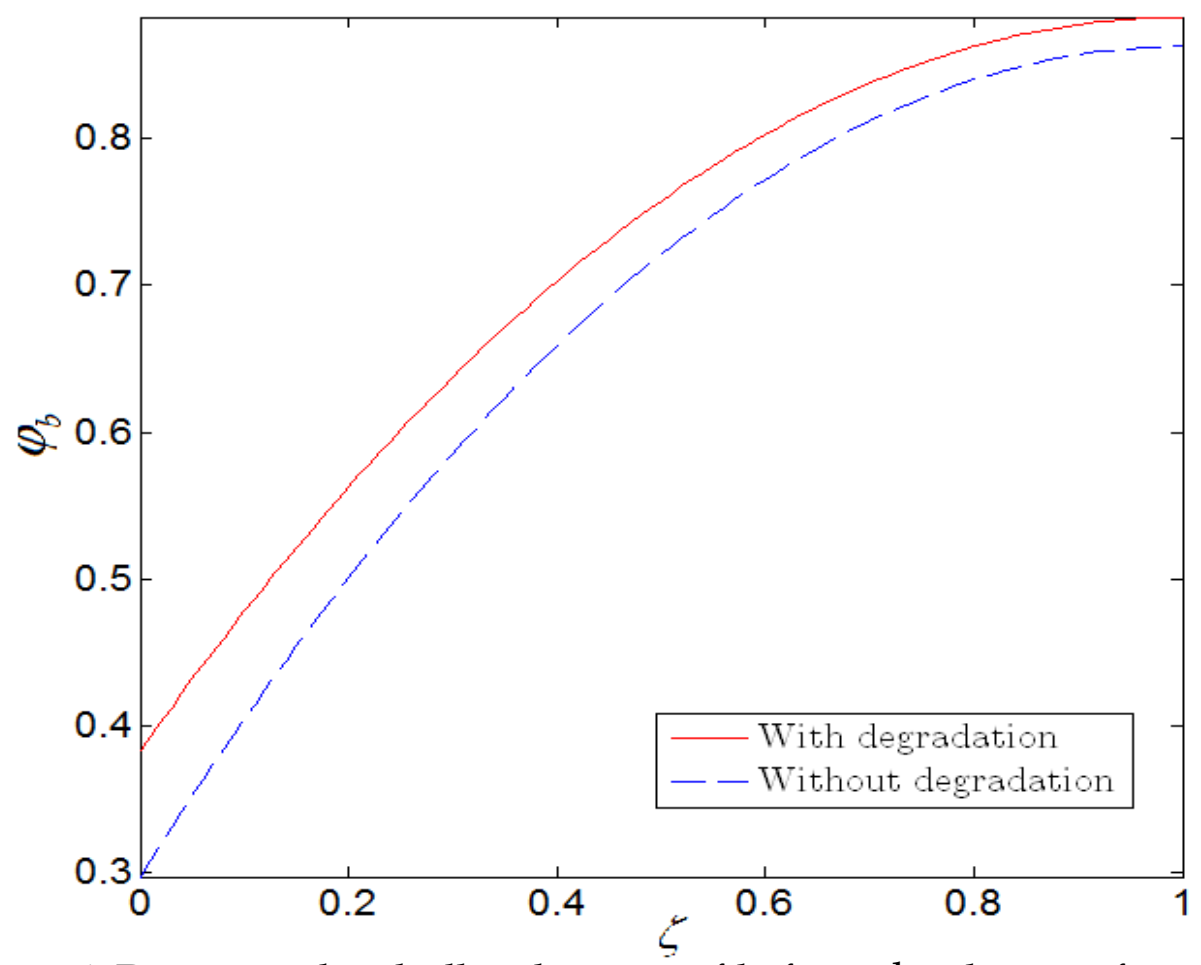

Figure 6: Dimensionless bulk velocity profile from the slip interface to the midplane of the bulk flow 
Table 1: Dimensional variables

\begin{tabular}{|c|c|c|c|}
\hline Name & Symbol & Dimensions & Range \\
\hline Absolute temperature & $T$ & $T$ & $>0$ \\
\hline Absolute temperature, bulk & $T_{b}$ & $T$ & $\geq 0$ \\
\hline Absolute temperature, drool & $T_{d}$ & $T$ & $\geq 0$ \\
\hline Absolute temperature, initial & $T_{0}$ & $T$ & $\geq 0$ \\
\hline Absolute temperature, interface (steady state) & $T_{i, \infty}$ & $T$ & $\geq 0$ \\
\hline Absolute temperature, melt degradation & $T_{D}$ & $T$ & $\geq 0$ \\
\hline Absolute temperature, wall & $T_{w}$ & $T$ & $\geq 0$ \\
\hline Activation energy & $E_{a}$ & $M L^{2} t^{-2} \mathrm{~mol}^{-1}$ & $\geq 0$ \\
\hline Arrhenius constant & $A$ & $t^{-1}$ & $\geq 0$ \\
\hline Binary diffusivity coefficient & $\mathscr{D}_{A B}$ & $L^{2} t^{-1}$ & $\geq 0$ \\
\hline Bulk fluid half-thickness & $b$ & $L$ & $\geq 0$ \\
\hline Calculated viscosity, material dependent & $\mu$ & $M L^{-1} t^{-1}$ & $\geq 0$ \\
\hline Channel depth* & $z$ & $L$ & $\geq 0$ \\
\hline Cohesive fracture strength & $\tau_{c}$ & $M L^{-1} t^{-2}$ & $\geq 0$ \\
\hline Density & $\rho$ & $M L^{-3}$ & $\geq 0$ \\
\hline Distance from die entrance & $y$ & $L$ & $\geq 0$ \\
\hline Distance from slip interface & $x$ & $L$ & $d \leq x \leq b$ \\
\hline Drool layer half-thickness & $d$ & $L$ & $\geq 0$ \\
\hline Final slit pressure, absolute & $p$ & $M L^{-1} t^{-2}$ & $\geq 0$ \\
\hline First order rate constant & $k$ & $t^{-1}$ & $\geq 0$ \\
\hline Fluid velocity, bulk flow parallel to die surface & $v_{b, y}(x)$ & $L t^{-1}$ & $\geq 0$ \\
\hline $\begin{array}{l}\text { Fluid velocity, drool flow parallel to die } \\
\text { surface }\end{array}$ & $v_{d, y}(x)$ & $L t^{-1}$ & $\geq 0$ \\
\hline Gas constant & $R$ & $M L^{2} t^{-2} \mathrm{~mol}^{-1} T$ & $>0$ \\
\hline Initial slit pressure, absolute & $p_{0}$ & $M L^{-1} t^{-2}$ & $\geq 0$ \\
\hline
\end{tabular}




\begin{tabular}{|l|c|c|c|}
\hline Inlet degraded polymer concentration & $c_{B 0}$ & $\mathrm{molL}^{-3}$ & $\geq 0$ \\
\hline Interfacial slip velocity & $v_{s}$ & $L t^{-1}$ & $\geq 0$ \\
\hline Intrinsic viscosity & {$[\eta]$} & $L M^{-1}$ & $\geq 0$ \\
\hline $\begin{array}{l}\text { Material dependent constant of } \\
\text { proportionality for viscosity [Eq. } \\
\text { Error! Reference source not found.] }\end{array}$ & $K$ & $\mathrm{ML}^{-1} \mathrm{t}^{-1}$ & $\geq 0$ \\
\hline Molar concentration of degradation product B & $c_{B}$ & $\mathrm{molL}^{-3}$ & $\geq 0$ \\
\hline Molar concentration of polymer A & $c_{A}$ & $\mathrm{molL}^{-3}$ & $>0$ \\
\hline Molecular weight, 2nd moment & $M_{\mathrm{w}}$ & $M \mathrm{~mol}^{-1}$ & $\geq 0$ \\
\hline Pressure differential & $\Delta P$ & $M L^{-1} t^{-2}$ & $\geq 0$ \\
\hline Rate of reaction (mass) & $r_{A}$ & $M t^{-1}$ & $\geq 0$ \\
\hline Relational viscosity, unrelated to material & $\mu_{0}$ & $M L^{-1} t^{-1}$ & $\geq 0$ \\
\hline Shear stress & $\tau_{y x}$ & $L t^{-1}$ & $\geq 0$ \\
\hline Slip heating flux & $q_{x}$ & $\mathrm{Mt}^{-3}$ & $\geq 0$ \\
\hline Slip velocity & $v_{s}$ & $L t^{-1}$ & $\geq 0$ \\
\hline Slit height & $h$ & $L$ & $\geq 0$ \\
\hline Slit length & $L$ & $L$ & $\geq 0$ \\
\hline Time on-stream & $t_{x}(y)$ & $t$ & $\geq 0$ \\
\hline
\end{tabular}


Table 2: Dimensionless variables.

\begin{tabular}{|c|c|c|c|}
\hline Name & Symbol & Definition & Range \\
\hline $\begin{array}{c}\text { Absolute } \\
\text { temperature, bulk } \\
\text { fluid }\end{array}$ & $\Theta_{d, \infty}$ & $\frac{\mathrm{T}_{\mathrm{b}}-\mathrm{T}_{0}}{\mathrm{~T}_{0}}$ & $\geq 0$ \\
\hline $\begin{array}{c}\text { Absolute } \\
\text { temperature, drool } \\
\text { layer }\end{array}$ & $\Theta_{b, \infty}$ & $\frac{\mathrm{T}_{\mathrm{d}}-\mathrm{T}_{0}}{\mathrm{~T}_{0}}$ & $\geq 0$ \\
\hline $\begin{array}{c}\text { Absolute } \\
\text { temperature, wall }\end{array}$ & $\Theta_{w}$ & $\frac{\mathrm{T}_{w}-\mathrm{T}_{0}}{\mathrm{~T}_{0}}$ & $\geq 0$ \\
\hline Activation energy & $s$ & $\frac{E_{a}}{R T_{0}}$ & $\geq 0$ \\
\hline $\begin{array}{l}\text { Arrhenius } \\
\text { coefficient }\end{array}$ & $r$ & $\frac{\epsilon}{e^{-E_{a} / R T}}=\frac{A \mu_{\mathrm{w}} L^{2}}{b^{2} \Delta P}$ & $\geq 0$ \\
\hline Buildup ratio & $B R$ & $\frac{Q_{d}}{Q_{b}}$ & $\geq 0$ \\
\hline Bulk flow rate* & $Q_{b}$ & $\frac{\dot{\mathrm{V}}_{\mathrm{b}} \mu_{\mathrm{w}} \mathrm{L}}{\mathrm{b}^{4} \Delta \mathrm{P}}$ & $\geq 0$ \\
\hline $\begin{array}{l}\text { Cohesive fracture } \\
\text { strength }\end{array}$ & $\sigma_{C}$ & $\frac{\tau_{C} L}{b \Delta P}$ & $\geq 0$ \\
\hline Die drool flow rate* & $Q_{d}$ & $\frac{\dot{\mathrm{V}}_{\mathrm{d}} \mu_{\mathrm{w}} \mathrm{L}}{\mathrm{b}^{4} \Delta \mathrm{P}}$ & $\geq 0$ \\
\hline $\begin{array}{l}\text { Distance down flow } \\
\text { field, parallel to the } \\
\text { length }\end{array}$ & 5 & $\frac{y}{L}$ & $0 \leq \varsigma \leq 1$ \\
\hline $\begin{array}{l}\text { Distance from slip } \\
\text { interface }\end{array}$ & $\zeta$ & $\frac{x}{b}$ & $-a \leq \zeta \leq 1$ \\
\hline $\begin{array}{l}\text { Drool layer } \\
\text { thickness }\end{array}$ & $a$ & $\frac{d}{b}$ & $\geq 0$ \\
\hline Feed resin purity & $g$ & $\frac{c_{B 0}}{c_{A 0}}$ & $2 \leq g \leq 0$ \\
\hline
\end{tabular}




\begin{tabular}{|c|c|c|c|}
\hline Interfacial heat flux & $F$ & $\frac{\mathrm{q}_{\mathrm{i}} \mathrm{b}}{\mathrm{kT}_{0}}$ & $\geq 0$ \\
\hline Kinetic rate constant & $\epsilon$ & $\frac{k \mu L^{2}}{b^{2} \Delta P}=\frac{A e^{\left(-\frac{E_{a}}{R T}\right)} \mu_{W} L^{2}}{b^{2} \Delta P}$ & $\geq 0$ \\
\hline $\begin{array}{l}\text { Mass fraction of } \\
\text { species A }\end{array}$ & $\omega_{A}$ & $\frac{c_{A}}{c}$ & $\geq 0$ \\
\hline $\begin{array}{l}\text { Material dependent } \\
\text { exponential constant } \\
\text { for viscosity }\end{array}$ & $\alpha$ & - & $\geq 0$ \\
\hline Slip heating flux & $F$ & $\frac{q_{i} \mathrm{~b}}{k T_{0}}$ & $\geq 0$ \\
\hline Slit width & W & $\frac{w}{b}$ & $\geq 0$ \\
\hline $\begin{array}{c}\text { Steady state } \\
\text { viscosity at interface }\end{array}$ & $\eta_{i, \infty}$ & $\frac{\mu}{\mu_{\mathrm{w}}}$ & $\geq 0$ \\
\hline Velocity, bulk* & $\boldsymbol{\varphi}_{b}(\zeta)$ & $\frac{v_{b} \mu_{w} L}{b^{2} \Delta P}$ & $\geq 0$ \\
\hline Velocity, drool* & $\boldsymbol{\varphi}_{d}(\zeta)$ & $\frac{v_{d} \mu_{\mathrm{w}} \mathrm{L}}{\mathrm{b}^{2} \Delta \mathrm{P}}$ & $\geq 0$ \\
\hline Velocity, slip* & $\boldsymbol{\varphi}_{s}$ & $\frac{v_{s} \mu_{\mathrm{w}} \mathrm{L}}{\mathrm{b}^{2} \Delta \mathrm{P}}$ & $\geq 0$ \\
\hline $\begin{array}{c}\text { Velocity-kinetics } \\
\text { normalizer }\end{array}$ & $\mathrm{U}$ & $\frac{k \mu_{\mathrm{w}} L^{2}}{b^{2} \Delta P}$ & $\geq 0$ \\
\hline Viscosity* & $\breve{\eta}$ & $\frac{\mu}{\mu_{\mathrm{w}}}$ & $\geq 0$ \\
\hline Viscosity coefficient & C & $\frac{a}{\ln ^{2} \eta_{i, \infty}}\left[\frac{a-\sigma_{C} \ln \eta_{i, \infty}}{\eta_{i, \infty}}-a+\ln \eta_{i, \infty}\left(\sigma_{C}+a\right)\right]+\varphi_{S}$ & $\geq 0$ \\
\hline
\end{tabular}

Legend: $M \equiv$ mass; $L \equiv$ length; $t \equiv$ time; $T \equiv$ temperature; ${ }^{*}$ identifies the second iteration of dimensionless variables, introduced in this paper in Section $X$, to help account for concentration profiles associated with scission. 
Table 3: Literature relevant to die drool with degradation.

\begin{tabular}{|c|c|c|c|c|c|c|}
\hline & Material & Method & $\begin{array}{l}\text { Investigated } \\
\text { Phenomenon }\end{array}$ & $\begin{array}{l}\text { Resulting } \\
\text { variables }\end{array}$ & Geometry & [Ref] \\
\hline $\begin{array}{c}\text { Brachet et al. } \\
\text { (2008) }\end{array}$ & LDPE & EXP & $\mathrm{DD}_{\mathrm{F}}$ & - & u & [32] \\
\hline $\begin{array}{l}\text { Yevtushenko } \\
\text { and Kuciej } \\
(2010)\end{array}$ & SP & $\begin{array}{l}\mathrm{AN}, \\
\mathrm{COM}\end{array}$ & $\mathrm{SH}$ & $T(\zeta)$ & 0 & [33] \\
\hline $\begin{array}{c}\text { Musil and } \\
\text { Zatloukal } \\
(2010,2012)\end{array}$ & HDPE & EXP & $\mathrm{DD}_{\mathrm{F}}$ & - & - & {$[11,21]$} \\
\hline $\begin{array}{l}\text { Schmalzer } \\
\text { (2013); } \\
\text { Schmalzer } \\
\text { and } \\
\text { Giacomin } \\
(2013,2014)\end{array}$ & AM & AN & $\mathrm{DD}_{\mathrm{CS}}$ & $B R$ & $\square, \odot$ & $\begin{array}{c}{[1,15,34,} \\
35,36]\end{array}$ \\
\hline $\begin{array}{l}\text { Gilbert and } \\
\text { Giacomin } \\
\text { (2014); } \\
\text { Gilbert } \\
(2014)\end{array}$ & NN & AN & $\mathrm{DD}_{\mathrm{CS}}, \mathrm{SH}$ & $\begin{array}{c}\Theta(\zeta) \\
Q_{(d)}, Q_{(b)}\end{array}$ & $\square$ & [16] \\
\hline $\begin{array}{l}\text { Gilbert } \\
\text { (2014); } \\
\text { Gilbert and } \\
\text { Giacomin } \\
\text { (2014); }\end{array}$ & NN & AN & $\begin{array}{c}\mathrm{DD}_{\mathrm{CS}}, \mathrm{VD}, \\
\mathrm{SH}\end{array}$ & $\begin{array}{c}\Theta(\zeta) \\
Q_{(d)}, Q_{(b)}\end{array}$ & $\square$ & $\begin{array}{c}\text { [19], [18], } \\
\text { [37] }\end{array}$ \\
\hline $\begin{array}{c}\text { Rorrer and } \\
\text { Dorgan } \\
(2014)\end{array}$ & $\begin{array}{c}\text { HDPE, } \\
\text { AM }\end{array}$ & $\mathrm{COM}$ & $\mathrm{DD}_{\mathrm{F}}$ & $\begin{array}{l}c_{B}(x, y), \\
\rho, v_{y}(x)\end{array}$ & $\square$ & [13] \\
\hline This paper & NN & AN & $\mathrm{SH}, \mathrm{DD}_{\mathrm{cs}}$ & $\Upsilon_{B}(\xi, \zeta)$ & $\square$ & \\
\hline
\end{tabular}

Legend: $\mathrm{NN} \equiv$ Newtonian, $\mathrm{AM} \equiv$ All materials, $\mathrm{SP} \equiv$ Solid pad; $\mathrm{AN} \equiv$ analytical, $\mathrm{COM} \equiv$ computational, $\mathrm{EXP} \equiv$ experimental; $\mathrm{DD}_{\mathrm{F}} \equiv$ die drool in fractionation paradigm, $\mathrm{DD}_{\mathrm{CS}} \equiv$ die drool in cohesive slip paradigm, $\mathrm{VD} \equiv$ viscous dissipation, $\mathrm{SH} \equiv$ slip heating; $B R \equiv$ buildup ratio, $\Theta \equiv$ adimensionalized temperature rise, $\zeta$ $\equiv$ adimensionalized distance from wall, $Q_{(d)} \equiv$ die drool flow rate, $Q_{(b)} \equiv$ bulk fluid flow rate, $T \equiv$ temperature profile, $c_{B}(x, y) \equiv$ spatial concentration, $\Upsilon_{B} \equiv$ adimensionalized degradate concentration, $\rho \equiv$ melt density; $\square \equiv$ slit flow, $\mathrm{U} \equiv$ blown film, $\odot \equiv$ pipe or tube; $\bullet \equiv$ screw extruder, $\circ \equiv$ parallel-disk. 


\section{REFERENCES}

${ }^{1}$ A.M. Schmalzer and A.J. Giacomin, "Die drool and die drool theory," Novel Trends in Rheology V, 1526, 35-46 (2013).

${ }^{2}$ F. Ding and A.J. Giacomin, "Die lines in plastics extrusion," Journal of Polymer Engineering, 20, (1), 1-39 (2000).

${ }^{3}$ J.M. Dealy and J. Wang, Melt rheology and its application in the plastics industry, 2nd ed., Springer, Munich (2013).

${ }^{4}$ J. Musil and M. Zatloukal, "Historical review of die drool phenomenon in plastics extrusion,” Polymer Reviews, 54, (1), 139-184 (Jan. 2014).

${ }^{5}$ A.J. Giacomin, J.D. Gander, and F. Ding, "Flaring dies to suppress die drool," Polymer Engineering and Science, 40(10), 2113-2123 (Oct. 2000).

${ }^{6}$ J. Musil and M. Zatloukal, "Effect of die exit geometry on internal die drool phenomenon during linear HDPE melt extrusion," International Journal of Heat and Mass Transfer, 56(1-2), 667-673 (Jan. 2013).

${ }^{7}$ C.-M. Chan, "Viscosity and the formation of die drool at the polymer-metal interfaces," International Polymer Processing, 10(3), 200-203 (Sep. 1995).

${ }^{8}$ T. Hogan, P. Walia, and B. Dems, "Investigation of the relationships between die build up and die swell," Polymer Engineering and Science, 49(2), 333-343 (2009).

${ }^{9}$ W.F. Busse, "Two decades of high-polymer physics: a survey and forecast," Physics Today, 17(9), 32-41 (1964).

${ }^{10}$ J.D. Gander and A.J. Giacomin, "Review of die lip build-up in plastics extrusion," Polymer Engineering and Science, 37(7), 1113-1126 (Jul. 1997).

${ }^{11}$ J. Musil and M. Zatloukal, "Investigation of die drool phenomenon for HDPE polymer melt," Chemical Engineering Science, 65, 6128-6133 (May 2010).

${ }^{12}$ H.P. Schreiber, "Component redistribution in extrusion of polyethylene compounds," Journal of Applied Polymer Science, 18(8), 2501-2508 (1974).

${ }^{13}$ N. Rorrer and J. Dorgan, "Molecular-scale simulation of cross-flow migration in polymer melts," Physical Review, 90(5), 1-10 (2014).

${ }^{14}$ M.J. MacDonald and S.J. Muller, "Experimental study of shear induced migration of polymers in dilute solutions," Journal of Rheology, 40(2), 259-283 (1996). 
15 A.M. Schmalzer and A.J. Giacomin, "Die drool theory," Journal of Polymer Engineering, 33(1), 1-18 (Feb. 2013).

${ }^{16}$ P.H. Gilbert and A.J. Giacomin, "Slip heating in die drool," PRG Report No. 001, QUCHEE-PRG-TR--2014-1, Polymers Research Group, Chemical Engineering Dept., Queen's University, Kingston, CANADA (March, 2014); Errata: In Eqs. (52) and (54), $W$ should be $2 W$.

${ }^{17}$ P.H. Gilbert and A.J. Giacomin, "Slip heating in die drool," The Canadian Journal of Chemical Engineering, DOI 10.1002/cjce.22148, 93, 580-589 (March 2015).

${ }^{18}$ P.H. Gilbert and A.J. Giacomin, "Slip heating in die drool with viscous dissipation," International Polymer Processing, DOI 10.3139/217.3002, (1), 141-146 (March 2015).

${ }^{19}$ P.H. Gilbert, "Slip heating in polymer processing," Masters Thesis, Chemical Engineering Department, Queen's University, Kingston, CANADA (November 27, 2014).

${ }^{20}$ R.B. Bird, W.E. Stewart, and E.N. Lightfoot, Transport phenomena, Revised 2nd ed. Wiley, New York (2007).

${ }^{21}$ J. Musil and M. Zatloukal, "Experimental investigation of flow induced molecular weight fractionation phenomenon for two linear HDPE polymer melts having same $M_{\mathrm{n}}$ and $M_{\mathrm{w}}$ but different $M_{\mathrm{z}}$ and $M_{\mathrm{z}+1}$ average molecular weights," Chemical Engineering Science, 81, 146-156 (Oct. 2012).

22 J.D. Gander, "Die lip build-up in plastics extrusion," Masters Thesis, University of Wisconsin, Mechanical Engineering Dept., Madison, WI (September, 1997).

${ }^{23}$ J.D. Gander and A.J. Giacomin, "Review of die lip build-up in plastics extrusion", S.P.E. Tech. Paper, XXXXII, Proc. 54th Annual Tech. Conf. \& Exhib., Society of Plastics Engineers, Indianapolis, IN (May, 1996), pp. 1113-1118.

${ }^{24}$ P. Atkins and J.D. Paula, Physical Chemistry, 2nd ed., Freeman, New York (2006).

${ }^{25}$ M. Zatloukal and K. Chaloupkova, "Effect of die design on die drool phenomenon for metallocene based 1ldpe: theoretical and experimental investigation," Journal of Applied Polymer Science, 111(10), 1728-1737 (2009).

${ }^{26}$ A.E. Hamielec, P.E. Gloor and S. Zhu, "Kinetics of free radical modification of polyolefins in extruders - chain scission, crosslinking and grafting," The Canadian Journal of Chemical Engineering, 69, 611-618 (Jun. 1991). 
${ }^{27}$ C. Beyler and M. Hirschler, "Thermal decomposition of polymers," in SFPE Handbook of Fire Protection Engineering, National Fire Protection Agency, Quincy, MA (2008), pp. 111-123.

${ }^{28}$ R. Zhao and C.W. Macosko, "Slip at polymer-polymer interfaces: rheological measurements on coextruded multilayers," Journal of Rheology, 46(1), 145-167 (2002).

${ }^{29}$ J. Fried, Polymer science and technology, 3rd ed., Prentice Hall, Upper Saddle River, NJ (2013).

${ }^{30}$ M. Schaffer, K. McAuley, E. Marchildon, and M. Cunningham, "Thermal degradation kinetics of nylon 66: experimental study and comparison with model predictions," Macromolecular Reaction Engineering, 1(5), 563-577 (2007).

${ }^{31}$ R.B. Bird, R.C. Armstrong and O. Hassager, Dynamics of polymeric liquids, vol. 1, 2nd ed., Wiley, New York (1987).

${ }^{32}$ P. Brachet, E.L. Hinrichsen, A. Larsen, L.K. Bjorndal, F. Brendryen, and T. Lia, "The problem of die drool in the extrusion of recycled polyethylene," Progress in Rubber Plastics and Recycling Technology, 25(4), 199-211 (Oct. 2008).

${ }^{33}$ A. Yevtushenko and M. Kuciej, "Two calculations schemes for determination of thermal stresses due to frictional heating during braking," Journal of Theoretical and Applied Mechanics, 48(3), 605-621 (2010).

${ }^{34}$ A.M. Schmalzer and A.J. Giacomin, "Slip between polyolefins and extrusion die surfaces," RRC Report No. 205, University of Wisconsin, Madison (May, 2013).

${ }^{35}$ A.M. Schmalzer and A.J. Giacomin, "A new dual-plate slipometer for measuring slip between molten polymers and extrusion die materials," Review of Scientific Instruments, 85, 045119 (2014).

${ }^{36}$ A.M. Schmalzer, "Slip between polyolefins and extrusion die surfaces," Masters Thesis, University of Wisconsin, Mechanical Engineering Dept., Madison, WI (June 11, 2013).

${ }^{37}$ P.H. Gilbert and A.J. Giacomin, "Slip heating in die drool with viscous dissipation," PRG Report No. 007, QU-CHEE-PRG-TR--2014-7, Polymers Research Group, Chemical Engineering Dept., Queen's University, Kingston, CANADA (July, 2014). 